CRYSTALLOGRAPHIC COMMUNICATIONS

ISSN 2056-9890

Received 22 July 2016

Accepted 26 July 2016

Edited by M. Zeller, Purdue University, USA

Keywords: crystal structure; ytterbium; amidinates; alkynylamidinate ligands; cyclopentadienyl complexes.

CCDC references: 1492937; 1492936

Supporting information: this article has supporting information at journals.iucr.org/e

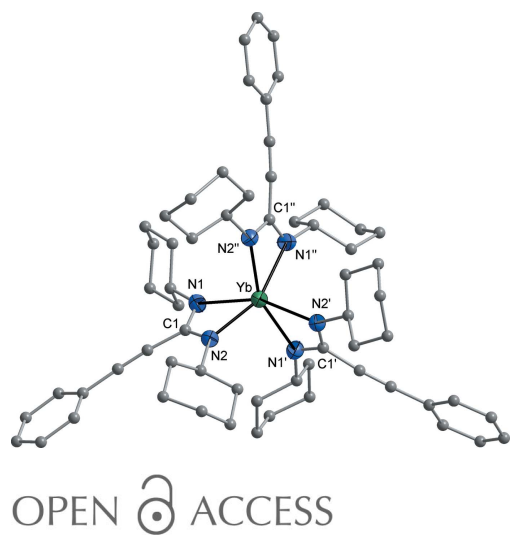

\section{Crystal structures of two ytterbium(III) complexes comprising alkynylamidinate ligands}

\author{
Sida Wang, ${ }^{a}$ Farid M. Sroor, ${ }^{\mathrm{b}}$ Phil Liebing, ${ }^{\mathrm{a}}$ Volker Lorenz, ${ }^{\mathrm{a}}$ Liane Hilfert ${ }^{\mathrm{a}}$ and \\ Frank T. Edelmann ${ }^{\mathrm{a} *}$ \\ ${ }^{a}$ Chemisches Institut der Otto-von-Guericke-Universitaet Magdeburg, Universitaetsplatz 2, 39106 Magdeburg, Germany, \\ and ${ }^{\mathbf{b}}$ Organometallic and Organometalloid Chemistry Department, National Research, Centre, 12622 Cairo, Egypt. \\ *Correspondence e-mail: frank.edelmann@ovgu.de
}

Two ytterbium(III) complexes comprising alkynylamidinate ligands, namely bis $\left(\eta^{5}\right.$-cyclopentadienyl)(3-cyclopropyl- $N, N^{\prime}$-diisopropylpropynamidinato$\left.\kappa^{2} N, N^{\prime}\right)$ ytterbium(III), [Y $\left.\mathrm{Yb}\left(\mathrm{C}_{5} \mathrm{H}_{5}\right)_{2}\left(\mathrm{C}_{12} \mathrm{H}_{19} \mathrm{~N}_{2}\right)\right]$ or $\mathrm{Cp}_{2} \mathrm{Yb}\left[\left({ }^{i} \operatorname{Pr}_{2} \mathrm{~N}\right)_{2} \mathrm{C}-\mathrm{C} \equiv \mathrm{C}-\right.$ $\left.c-\mathrm{C}_{3} \mathrm{H}_{5}\right]$ (1) and tris(3-phenyl- $N, N^{\prime}$-dicyclohexylpropynamidinato- $\left.\kappa^{2} N, N^{\prime}\right)$ ytterbium(III), $\left[\mathrm{Yb}\left(\mathrm{C}_{21} \mathrm{H}_{27} \mathrm{~N}_{2}\right)_{3}\right]$ or $\mathrm{Yb}\left[(\mathrm{CyN})_{2} \mathrm{C}-\mathrm{C} \equiv \mathrm{C}-\mathrm{Ph}\right]_{3}(\mathrm{Cy}=$ cyclohexyl) (2) have been synthesized and structurally characterized. Both complexes are monomers; for complex $\mathbf{2}$, the contribution to the scattering from highly disordered toluene solvent molecules in these voids was removed with the SQUEEZE routine [Spek (2015). Acta Cryst. C71, 9-18] in PLATON. The stated crystal data for $M r, \mu$ etc. do not take these into account.

\section{Chemical context}

Anionic amidinate ligands of the type $\left[R \mathrm{C}\left(\mathrm{N} R^{\prime}\right)_{2}\right]^{-}(R=\mathrm{H}$, alkyl, aryl; $R^{\prime}=$ alkyl, cycloalkyl, aryl, $\left.\mathrm{SiMe}_{3}\right)$ are highly useful and versatile spectator ligands in organolanthanide chemistry. These readily available $N$-chelating ligands are generally regarded as sterically demanding cyclopentadienyl equivalents (Collins, 2011; Edelmann, 2013). Mono-, di- and trisubstituted lanthanide amidinate complexes are all accessible, in close analogy to the long known mono-, di- and tricyclopentadienyl complexes. Over the past $c a 25$ years, lanthanide amidinates have witnessed an impressive transformation from laboratory curiosities to homogeneous catalysts as well as valuable precursors in materials science. Rare-earth metal amidinates have been reported to be highly active homogeneous catalysts e.g. for ring-opening polymerization reactions of lactones, the guanylation of amines or the addition of terminal alkynes to carbodiimides (Edelmann, 2009, 2012). In materials science, certain homoleptic alkyl-substituted lanthanide tris(amidinate) complexes are highly volatile and can be used as precursors for ALD (atomic layer deposition) and MOCVD (metal-organic chemical vapor deposition) processes, e.g. for the deposition of lanthanide oxide $\left(\mathrm{Ln}_{2} \mathrm{O}_{3}\right)$ or lanthanide nitride $(\operatorname{LnN})$ thin films (Devi, 2013).

Introduction of alkynyl groups to the central $\mathrm{C}$ atom in amidines provides alkynylamidines of the general type $R-$ $\mathrm{C} \equiv \mathrm{C}-\mathrm{C}\left(\mathrm{N} R^{\prime}\right)\left(\mathrm{NH} R^{\prime}\right)$. In organic synthesis, alkynylamidines have been frequently employed in the preparation of various heterocycles (Ong et al., 2006; Xu et al., 2008; Weingärtner \& Maas, 2012). Alkynylamidines are also useful for diverse applications in biological and pharmacological systems (Rowley et al., 2005; Sienkiewicz et al., 2005). Thus far, only a 
few lanthanide complexes containing alkynylamidinate ligands have been described. Previously used alkynylamidinate ligands include e.g. phenylethynyl derivatives $[\mathrm{Ph}-$ $\left.\mathrm{C} \equiv \mathrm{C}-\mathrm{C}(\mathrm{N} R)_{2}\right]^{-}\left(R={ }^{i} \mathrm{Pr},{ }^{t} \mathrm{Bu}\right)$ (Dröse et al., 2010a,b; $\mathrm{Xu}$ et al., 2013) and the trimethylsilyl-substituted anions $\left[\mathrm{Me}_{3} \mathrm{Si}-\right.$ $\left.\mathrm{C} \equiv \mathrm{C}-\mathrm{C}(\mathrm{N} R)_{2}\right]^{-}\left[R=\right.$ cyclohexyl $\left.(\mathrm{Cy}),{ }^{i} \mathrm{Pr}\right]$ (Seidel et al., 2012).

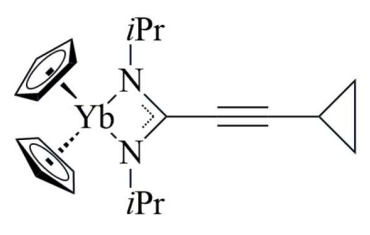

1

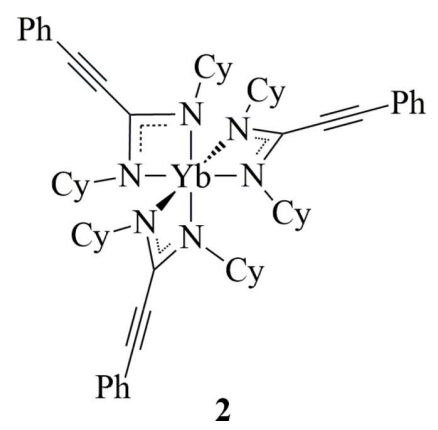

2
We recently initiated a study of alkynylamidinates derived from cyclopropylacetylene (Sroor et al., 2015c). The cyclopropyl group was chosen because of the well-known electrondonating ability of this substituent to an adjacent electrondeficient atom or group. This would give us the rare chance to electronically influence the amidinate ligand system rather than altering only its steric demand. We now describe the synthesis and structural characterization of two new ytterbium(III) alkynylamidinate complexes, namely $\mathrm{Cp}_{2} \mathrm{Yb}\left[\left({ }^{i} \mathrm{PrN}\right)_{2} \mathrm{C}-\mathrm{C} \equiv \mathrm{C}-c-\mathrm{C}_{3} \mathrm{H}_{5}\right] \quad$ (1) and $\mathrm{Yb}\left[(\mathrm{CyN})_{2} \mathrm{C}-\right.$ $\mathrm{C} \equiv \mathrm{C}-\mathrm{Ph}]_{3}(\mathrm{Cy}=$ cyclohexyl; 2), shown in Figs. 1 and 2.

\section{Structural commentary}

The structural analyses revealed that both title compounds are monomeric in the solid state, with the alkynylamidinate anion acting as an $N, N^{\prime}$-chelating ligand. Compound $\mathbf{1}$ crystallizes in the orthorhombic space group $\mathrm{Pbca}$ with one complex molecule in the asymmetric unit. The two cyclopentadienyl ligands feature a typical symmetric $\eta^{5}$-coordination with $\mathrm{Yb}-$

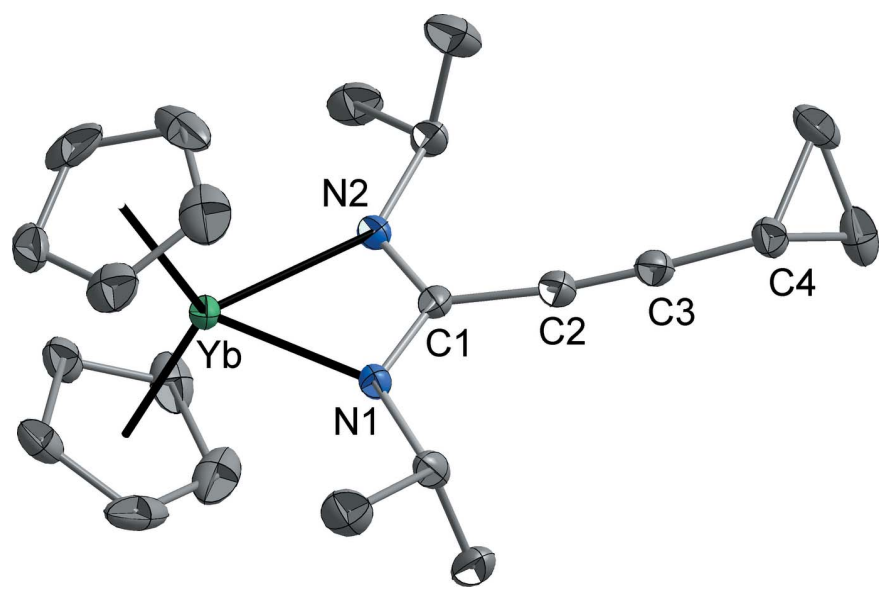

Figure 1

The molecular structure of compound $\mathbf{1}$.

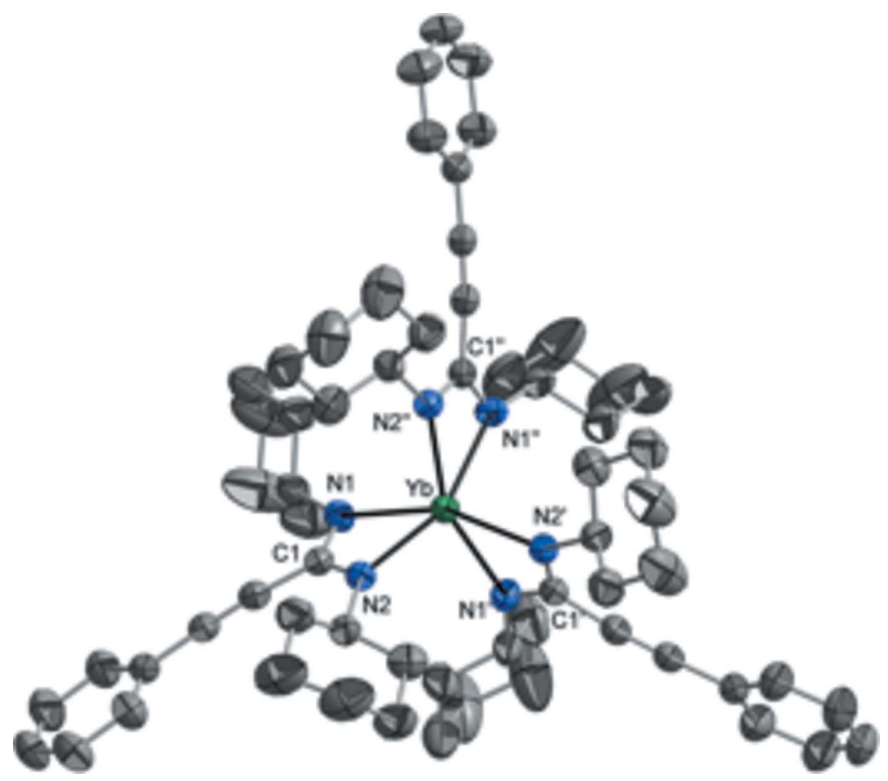

Figure 2

The molecular structure of compound 2. Displacement ellipsoids are at the $50 \%$ probability level and $\mathrm{H}$ atoms have been omitted for clarity. Only one orientation of the disordered cyclohexyl group at N2 is shown. The $\mathrm{Yb}$ atom is located on a threefold rotation axis parallel to the crystallographic $c$ axis. [Symmetry operators to generate equivalent atoms: (') $1-y,-1+x-y, z$; (') $2-x+y, 1-x, z$.]

centroid(Cp) distances of 2.315 and $2.321 \AA$. The $\mathrm{Yb}-\mathrm{Cp}$ distances are therefore slightly larger than in the related chloride $\left[\mathrm{Cp}_{2} \mathrm{YbCl}\right]_{2}[\mathrm{Yb}-$ centroid(Cp) 2.300 and $2.307 \AA$; Lamberts et al., 1987; Lueken et al., 1987, 1989], possibly due to the steric demand of the two $N$-isopropyl groups close to the ytterbium atom. Probably for the same reason, the product does not contain coordinating THF even though the complex was prepared in THF solution. Accordingly the coordination geometry around $\mathrm{Yb}$ can be described as distorted pseudotetrahedral. At $131.1^{\circ}$, the $\mathrm{Cp}-\mathrm{Yb}-\mathrm{Cp}$ angle is close to that observed in $\left[\mathrm{Cp}_{2} \mathrm{YbCl}_{2}\left(\mathrm{Cp}-\mathrm{Yb}-\mathrm{Cp} 130.0^{\circ}\right.\right.$; Lamberts et al., 1987; Lueken et al., 1987, 1989) and compound $\mathbf{1}$ is therefore a typical bent metallocene complex of trivalent ytterbium. Due to the low formal coordination number of four around the $\mathrm{Yb}$ atom, the $\mathrm{Yb}-\mathrm{N}$ bond lengths of 2.274 (2) and 2.293 (2) $\AA$ are short compared to those observed in other late lanthanide amidinates, such as $\left[\mathrm{Yb}_{2}\left\{(\mathrm{DippN})_{2} \mathrm{CH}\right\}_{4}\left(\mu-\mathrm{OCPh}=\mathrm{C}_{6} \mathrm{H}_{4}-4-\right.\right.$ $\left.\left.\mathrm{CPh}_{2} \mathrm{O}\right)(\mathrm{THF})\right][\mathrm{Yb}-\mathrm{N} 2.285$ (2)-2.391 (2) $\AA$; Deacon et al., 2014], $\left[\mathrm{Ho}\left\{\mathrm{N}\left(\mathrm{SiMe}_{3}\right)_{2}\right\}\left\{(\mathrm{CyN})_{2} \mathrm{C}-\mathrm{C} \equiv \mathrm{C}-c-\mathrm{C}_{3} \mathrm{H}_{5}\right\}_{2}\right][\mathrm{Ho}-\mathrm{N}$ 2.303 (2)-2.348 (4) $\AA$; Sroor et al., 2015b] and $\left[\mathrm{Ho}\left(\eta^{8}\right.\right.$ $\left.\mathrm{COT})\left\{(\mathrm{CyN})_{2} \mathrm{C}-\mathrm{C} \equiv \mathrm{C}-c-\mathrm{C}_{3} \mathrm{H}_{5}\right\}(\mathrm{THF})\right] \quad[\mathrm{Ho}-\mathrm{N} 2.342$ (3) and 2.349 (3) Аं; Sroor et al., 2016].

Compound 2 crystallizes in the trigonal space group $R \overline{3} c$, with the $\mathrm{Yb}$ atom located on a threefold rotation axis along the crystallographic $c$ axis. The complex molecule is therefore C3 symmetric. The $\mathrm{Yb}$ atom is coordinated by the three symmetry-equivalent chelating amidinate ligands in a distorted octahedral fashion with $\mathrm{C} 1-\mathrm{Yb}-\mathrm{Cl}^{\prime}$ angles of $120^{\circ}$ and an angle of $90 \pm 3^{\circ}$ between the $\mathrm{YbN}_{2} \mathrm{C}$ planes. The cyclohexyl group attached to $\mathrm{N} 2$ is disordered over two 
orientations by rotation around the $\mathrm{N} 2-\mathrm{C} 16$ vector. As a result of the higher coordination number, the $\mathrm{Yb}-\mathrm{N}$ bonds [2.310 (2) and 2.320(2) $\mathrm{A}]$ are slightly longer than in compound $\mathbf{1}$. However, in consequence of the small size of the $\mathrm{Yb}^{3+}$ ion, the $\mathrm{Yb}-\mathrm{N}$ bonds in compound $\mathbf{2}$ are significantly shorter than in corresponding hexacoordinated lanthanide(III) amidinates, e.g. $\left[\operatorname{Ln}\left\{\left({ }^{i} \mathrm{PrN}\right)_{2} \mathrm{C}-{ }^{t} \mathrm{Bu}\right\}_{3}\right][\operatorname{Ln}=\mathrm{Ce}: \mathrm{Ce}-\mathrm{N}$ 2.469 (2)-2.550 (2) $\AA$; $L n=\mathrm{Eu}: \mathrm{Eu}-\mathrm{N} 2.402$ (4)-2.457 (4) $\AA$; $L n=\mathrm{Tb}: \mathrm{Tb}-\mathrm{N} 2.391$ (3)-2.409 (3) $\AA$; Dröse et al., 2011] and $\left[\mathrm{Ho}\left\{(\mathrm{CyN})_{2} \mathrm{C}-\mathrm{C} \equiv \mathrm{C}-c-\mathrm{C}_{3} \mathrm{H}_{5}\right\}_{3}\right] \quad[\mathrm{Ho}-\mathrm{N} \quad 2.342(2)-$ 2.383 (3) $\mathrm{A}]$ (Sroor et al., 2015a).

The $\mathrm{N}-\mathrm{Yb}-\mathrm{N}$ angle in compound $2\left[58.2(1)^{\circ}\right]$ is slightly smaller than in compound $\mathbf{1}\left[59.1(1)^{\circ}\right]$, but larger than in other homoleptic lanthanide (III) amidinates $\{$ e.g. $\left[\operatorname{Ln}\left\{\left({ }^{i} \operatorname{PrN}\right)_{2} \mathrm{C}-{ }^{t} \mathrm{Bu}\right\}_{3}\right], \operatorname{Ln}=\mathrm{Ce}: \mathrm{N}-\mathrm{Ce}-\mathrm{N} 51.81(4)-52.72(4)^{\circ}$; $L n=\mathrm{Eu}: \mathrm{N}-\mathrm{Eu}-\mathrm{N} 53.9(1)-54.4(2)^{\circ} ; L n=\mathrm{Tb}: \mathrm{N}-\mathrm{Tb}-\mathrm{N}$ 54.9 (1)-55.0 (1) ; Dröse et al., 2011\} and $\left[\mathrm{Ho}\left\{(\mathrm{CyN})_{2} \mathrm{C}-\right.\right.$ $\left.\left.\mathrm{C} \equiv \mathrm{C}-c-\mathrm{C}_{3} \mathrm{H}_{5}\right\}_{3}\right]\left[\mathrm{N}-\mathrm{Ho}-\mathrm{N} 57.1\right.$ (1)-57.7 (1) ${ }^{\circ}$; Sroor et al., 2015a]. The $\mathrm{N}-L n-\mathrm{N}$ angle therefore correlates clearly with the $L n-\mathrm{N}$ bond length, decreasing with rising $L n-\mathrm{N}$ distance (i.e. with rising coordination number of the metal and within the lanthanide series from right to left). The $\mathrm{C} 1-\mathrm{N}$ bond lengths of the amidinate ligand are very similar [1: 1.332 (3) and 1.334 (3) $\AA$; 2: 1.321 (4) and 1.324 (4) ] ], indicating a typical delocalization of the negative charge within the NCN fragment (Sroor et al., 2016).

\section{Supramolecular features}

Compounds $\mathbf{1}$ and $\mathbf{2}$ do not exhibit any specific intermolecular interactions. In compound $\mathbf{1}$, the closest intermolecular $\mathrm{C}-\mathrm{C}$ contacts are found between $\mathrm{Cp}$ ligands and cyclopropyl substituents, 3.510-3.625 ̊̊. Compound 2 features one intermolecular phenyl-cyclohexyl contact where the shortest $\mathrm{C}-\mathrm{C}$ distance is $3.567 \AA$, and various cyclohexyl-cyclohexyl contacts with $\mathrm{C}-\mathrm{C}$ distances of 3.441-3.576 $\mathrm{\AA}$. The crystal structure of compound 2 comprises a large void of $c a 220 \AA^{3}$ that is probably filled with a highly disordered toluene molecule. The content of the voids was corrected for using the SQUEEZE method (Spek, 2015), yielding a solvent-accessible volume of $1316 \AA^{3}$ and 138 electrons, or about 1.5 solvate molecules per unit cell. The composition of the crystal can therefore be assumed to be $\mathbf{2} \cdot 0.166$ toluene.

\section{Database survey}

For other lanthanide(III) complexes with amidinate ligands, see Richter et al. (2004), Edelmann $(2009,2012)$ and Deacon et al. (2014). For related bent sandwich complexes of the lanthanides, see Lueken et al. (1987, 1989), Schumann et al. (1998) and Kühling et al. (2015).

\section{Synthesis and crystallization}

\section{Synthesis of $\mathrm{Cp}_{2} \mathbf{Y b}\left[\left({ }^{i} \mathrm{Pr}_{2} \mathrm{~N}\right)_{2} \mathrm{C}-\mathrm{C} \equiv \mathrm{C}-c-\mathrm{C}_{3} \mathrm{H}_{5}\right]$ (1)}

This compound was prepared by treatment of $\mathrm{Cp}_{2} \mathrm{YbCl}$ (Maginn et al., 1963) with $\mathrm{Li}\left[\left({ }^{i} \mathrm{Pr}_{2} \mathrm{~N}\right)_{2} \mathrm{C}-\mathrm{C} \equiv \mathrm{C}-c-\mathrm{C}_{3} \mathrm{H}_{5}\right]$
(Sroor et al., 2013) in a molar ratio of 1:1. Treatment of $\mathrm{Cp}_{2} \mathrm{YbCl} \quad(0.68 \mathrm{~g}, 2.0 \mathrm{mmol})$ with $\operatorname{Li}\left[\left({ }^{i} \operatorname{Pr}_{2} \mathrm{~N}\right)_{2} \mathrm{C}-\mathrm{C} \equiv \mathrm{C}-\right.$ $\left.c-\mathrm{C}_{3} \mathrm{H}_{5}\right]\left(2.0 \mathrm{mmol}\right.$, prepared in situ from $\mathrm{Li}-\mathrm{C} \equiv \mathrm{C}-c-\mathrm{C}_{3} \mathrm{H}_{5}$ and $N, N^{\prime}$-diisopropylcarbodiimide) in $30 \mathrm{ml}$ of THF produced a bright-orange solution and a white precipitate $(\mathrm{LiCl})$. After filtration and evaporation to dryness, the product was extracted with $n$-pentane $(2 \times 20 \mathrm{ml})$. The extract was filtered again and concentrated to a total volume of $c a 10 \mathrm{ml}$. Crystallization at $253 \mathrm{~K}$ afforded $\mathbf{1}$ as orange air- and moisturesensitive crystals. Yield: 0.53 g, 73\%. M.p.: $478 \mathrm{~K}$. Analysis calculated for $\mathrm{C}_{22} \mathrm{H}_{20} \mathrm{~N}_{2} \mathrm{Yb}$ : C 53.43, $\mathrm{H} 5.91, \mathrm{~N} 5.66 \%$; found: $\mathrm{C}$ 53.61, H 5.766, N 5.86\%. MS (EI, $M=494.54): \mathrm{m} / z$ (\%) 450 (5) $\left[M-3 \mathrm{CH}_{3}\right]^{+}, 407(5)\left[M-2^{i} \mathrm{Pr}\right]^{+}, 384$ (7), 369 (13) $[M-2 \mathrm{Cp}$ $+3 \mathrm{H}]^{+}, 355(5), 341(66)\left[M-\mathrm{Cp}-2^{i} \mathrm{Pr}\right]^{+}, 328$ (5), 313 (4) $\left[\mathrm{YbN}{ }^{i} \mathrm{Pr}-\mathrm{C}(\mathrm{CH})-\mathrm{N}^{i} \mathrm{Pr}\right]^{+}, \quad 299(7) \quad\left[\mathrm{YbN}{ }^{i} \mathrm{Pr}-\mathrm{C}-\mathrm{N}^{i} \mathrm{Pr}\right]^{+}$, 284 (10) $\left[\mathrm{YbN}^{i} \mathrm{Pr}-\mathrm{C}-\mathrm{NCCH}_{3}\right]^{+}, 274$ (100) $\left[\mathrm{YbN}^{i} \mathrm{Pr}-\mathrm{C}-\right.$ $\left.\mathrm{NCH}_{3}\right]^{+}, 258(25)\left[\mathrm{YbN}{ }^{i} \mathrm{Pr}-\mathrm{CN}\right]^{+}, 243$ (8), 232 (10), 215 (12) $[\mathrm{YbNCN}]^{+}$. IR $(\mathrm{KBr}) v\left(\mathrm{~cm}^{-1}\right): 3093(w), 2963(\mathrm{~m}), 2922(w)$, $2871(w), 2609(w), 2215(m, \mathrm{C} \equiv \mathrm{C}), 2070(w), 1985(w), 1746$ $(w), 1609(m, \mathrm{NCN}), 1450(\mathrm{~s}), 1367(\mathrm{~m}), 1327(\mathrm{~m}), 1258(w)$, $1224(\mathrm{~m}), 1177(\mathrm{~m}), 1055(\mathrm{w}), 1012(\mathrm{~m}), 968(\mathrm{~m}), 878(\mathrm{w}), 766$ $(v s), 695(m), 531(w), 481(w), 393(w), 328(w) .{ }^{1} \mathrm{H}$ NMR (400 MHz, [D 6 -benzene, $298 \mathrm{~K}$ ): $\delta 0.92$ (overlapped, $m, \mathrm{CH}$ cyclopropyl), $0.47-0.51$ ( $m, 2 \mathrm{H}, \mathrm{CH}_{2}$-cyclopropyl), $0.25-0.20$ ( $m, 2 \mathrm{H}, \mathrm{CH}_{2}$-cyclopropyl), $-1.5($ br s $, 10 \mathrm{H}, \mathrm{CH} \mathrm{Cp}),-7.2(1 \mathrm{H}$, sept, $\left.\mathrm{CH}{ }^{i} \mathrm{Pr}\right),-10.8\left(1 \mathrm{H}\right.$, sept, $\left.\mathrm{CH}{ }^{i} \mathrm{Pr}\right),-36.9$ (br s, $\left.\mathrm{CH}_{3}{ }^{i} \mathrm{Pr}\right)$. ${ }^{13} \mathrm{C}$ NMR (100.6 MHz, [D $\mathrm{D}_{6}$ ]-benzene, $\left.298 \mathrm{~K}\right): \delta 152.3(s, \mathrm{NCN})$, $96.4(s, C \equiv \mathrm{C}-\mathrm{C}), 69.7(s, C H-\mathrm{C} \equiv \mathrm{C}), 65.3(s, C H \mathrm{Cp}), 2.5-2.6$ ( $s, \mathrm{CH}^{i} \mathrm{Pr}$ ), 1.1 (br s, $\left.\mathrm{CH}_{3}{ }^{i} \mathrm{Pr}\right), 8.4$ ( $s, \mathrm{CH}_{2}$ cyclopropyl), -0.4 ( $s, \mathrm{CH}$ cyclopropyl). (2)

Synthesis of $\mathrm{Yb}\left[(\mathrm{CyN})_{2} \mathrm{C}-\mathrm{C} \equiv \mathrm{C}-\mathrm{Ph}\right]_{3}(\mathrm{Cy}=$ cyclohexyl $)$

Anhydrous ytterbium(III) trichloride $(1.40 \mathrm{~g}, 5.0 \mathrm{mmol})$ (Freeman \& Smith, 1958) was suspended in THF $(50 \mathrm{ml})$ and treated with a solution of $\mathrm{Li}\left[\mathrm{Ph}-\mathrm{C} \equiv \mathrm{C}-\mathrm{C}(\mathrm{NCy})_{2}\right](4.72 \mathrm{~g}$, $15.0 \mathrm{mmol}$ ) (prepared in situ by addition of lithium phenylacetylide to $N, N^{\prime}$-dicyclohexylcarbodiimide) in THF $(60 \mathrm{ml})$. The reaction mixture was refluxed for $3 \mathrm{~h}$. After cooling to room temperature, the white precipitate $(\mathrm{LiCl})$ was removed by filtration, and the clear filtrate was evaporated to dryness. Off-white air- and moisture-sensitive solid. Yield: $3.07 \mathrm{~g}, 56 \%$. M.p.: 505 K. Single crystal suitable for X-ray structure determination were obtained from a saturated toluene solution at $281 \mathrm{~K}$. Analysis calculated for $\mathrm{C}_{63} \mathrm{H}_{81} \mathrm{~N}_{6} \mathrm{Yb}$ : C 69.07, H 7.45, N 7.67\%; found: C 69.21, H 7.50, N 7.47\%. MS (EI, $M=1095.42$ ): $m / z(\%) 1014(23)[M-\mathrm{Cy}]^{+}, 1006$ (7) $\left[M-\mathrm{PhC}^{+}, 998(15)\right.$, 964 (14), 949 (16), 899 (46), 849 (30), 833 (20), 811 (12), 799 (23), 787 (75) $[M-\mathrm{NCy}-\mathrm{C}(\mathrm{C} \equiv \mathrm{C}-\mathrm{Ph})-\mathrm{NCy}]^{+}$, 783 (35), 733 (62), 711 (6) [M- NCy-C(C $\equiv \mathrm{C}-\mathrm{Ph})-\mathrm{NCy}-$ $\mathrm{Ph}]^{+}, 683$ (45), 667 (100) $[M-\mathrm{NCy}-\mathrm{C}(\mathrm{C} \equiv \mathrm{C}-\mathrm{Ph})-\mathrm{NCy}-$ $\left.\mathrm{Ph}-\mathrm{C}_{3} \mathrm{H}_{8}\right]^{+}, 645(29)$. IR (KBr) $v\left(\mathrm{~cm}^{-1}\right): 2922(\mathrm{~s}), 2850(\mathrm{~m})$, $2661(w), 2208$ ( $w, \mathrm{C}=\mathrm{C}), 1982(w), 1598(w), 1574(w, \mathrm{NCN})$, $1491(\mathrm{~m}), 1461(\mathrm{vs}), 1449(\mathrm{~s}), 1411(\mathrm{~m}), 1398(\mathrm{~m}), 1343(\mathrm{~s}), 1311$ $(\mathrm{m}), 1256(\mathrm{~m}), 1192(\mathrm{~m}), 1170(\mathrm{~m}), 1137(\mathrm{~m}), 1070(\mathrm{~m}), 1027$ (w), $995(m), 914(w), 898(m), 887(m), 844(w), 798(w), 754$ $(s), 702(m), 688(s), 628(s), 553(w), 529(m), 504(w), 488(w)$, $452(w), 411(m), 355(m), 316(m), 273(w) .{ }^{1} \mathrm{H}$ NMR 
Table 1

Experimental details.

1

Crystal data

Chemical formula

$M_{\text {r }}$

Crystal system, space group

Temperature $(\mathrm{K})$

$a, b, c(\AA)$

$\alpha, \beta, \gamma\left(^{\circ}\right)$

$V\left(\AA^{3}\right)$

$Z$

Radiation type

$\mu\left(\mathrm{mm}^{-1}\right)$

Crystal size (mm)

Data collection

Diffractometer

Absorption correction

$T_{\min }, T_{\max }$

No. of measured, independent and

observed $[I>2 \sigma(I)]$ reflections

$R_{\text {int }}$

$(\sin \theta / \lambda)_{\max }\left(\AA^{-1}\right)$

Refinement

$R\left[F^{2}>2 \sigma\left(F^{2}\right)\right], w R\left(F^{2}\right), S$

No. of reflections

No. of parameters

$\mathrm{H}$-atom treatment

$\Delta \rho_{\max }, \Delta \rho_{\min }\left(\mathrm{e} \AA^{-3}\right)$
$\left[\mathrm{Yb}\left(\mathrm{C}_{5} \mathrm{H}_{5}\right)_{2}\left(\mathrm{C}_{12} \mathrm{H}_{19} \mathrm{~N}_{2}\right)\right]$

494.51

Orthorhombic, $\mathrm{Pbca}$

153

9.4578 (2), 19.2910 (6), 22.2114 (5)

90, 90,90

4052.48 (18)

8

Mo $K \alpha$

4.62

$0.33 \times 0.31 \times 0.25$

Stoe IPDS 2T

Numerical $(X-A R E A$ and $X-R E D$; Stoe \& Cie, 2002)

$0.326,0.448$

$21905,4045,3263$

0.051

0.620

$0.018,0.040,0.98$

4045

227

$\mathrm{H}$-atom parameters constrained

$0.50,-0.72$
2

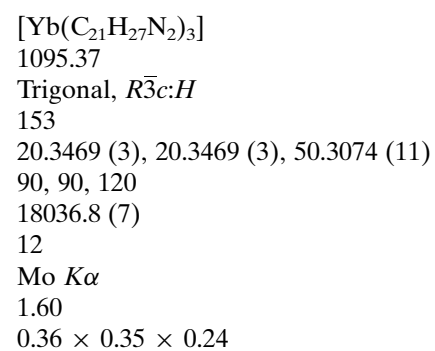

Stoe IPDS 2T

Numerical $(X-A R E A$ and $X$-RED; Stoe \& Cie, 2002)

$0.621,0.722$

$36832,3578,2774$

0.065

0.597

$0.029,0.071,1.07$

3578

257

$\mathrm{H}$-atom parameters constrained

$0.31,-1.18$

Computer programs: X-AREA and X-RED (Stoe \& Cie, 2002), SHELXS2013 (Sheldrick, 2008), SHELXL2014 (Sheldrick, 2015) and DIAMOND (Brandenburg, 1999).

(400.1 MHz, [D $\left.\mathrm{D}_{8}\right]$-THF, $\left.298 \mathrm{~K}\right): 14.12$ (br s, $\left.\mathrm{CH}_{2}, \mathrm{Cy}\right), 6.88$ (br $\left.s, \mathrm{CH}_{2}, \mathrm{Cy}\right), 4.54(m, 3 \mathrm{H}, p-\mathrm{CH} \mathrm{Ph}), 3.94(m, 6 \mathrm{H}, m-\mathrm{CH} \mathrm{Ph})$, 1.29 (brs $\left., \mathrm{CH}_{2}, \mathrm{Cy}\right),-0.15(\mathrm{~d}, 6 \mathrm{H}, o-\mathrm{CH} \mathrm{Ph}),-14.62$ (br s, N$\mathrm{CH}, \mathrm{Cy}) .{ }^{13} \mathrm{C}$ NMR $\left(100.6 \mathrm{MHz},\left[\mathrm{D}_{8}\right]-\mathrm{THF}, 298 \mathrm{~K}\right): 126.4$ ( $s, p-$ $C H \mathrm{Ph}), 126.0$ ( $s, m-C H \mathrm{Ph}), 124.6$ ( $s, o-C H \mathrm{Ph}), 111.2$ ( $s, i-C$ $\mathrm{Ph}$ ), $71.0(s, \equiv C$-Ph), 46.0 ( $s, \mathrm{~N}-\mathrm{CH}, \mathrm{Cy}), 36.1\left(\mathrm{~s}, \mathrm{CH}_{2}, \mathrm{Cy}\right)$, $35.7\left(s, \mathrm{CH}_{2}, \mathrm{Cy}\right), 35.0\left(s, \mathrm{CH}_{2}, \mathrm{Cy}\right), \equiv C-\mathrm{C}(\mathrm{NCy})_{2}$ and $\mathrm{NCN}$ not observed.

\section{Refinement}

Crystal data, data collection and structure refinement details are summarized in Table 1 . In the case of compound 2, C atoms C17-C21 of the disordered cyclohexyl substituent have been split over two sites, with a freely refined occupancy ratio. The N-bonded C atom C16 was refined as not disordered using EXYZ and EADP commands but the different orientation of the corresponding $\mathrm{H}$ atom $\mathrm{H} 17$ was taken into account. The contribution to the scattering from the solvent molecule in compound $\mathbf{2}$ was removed with the SQUEEZE routine (Spek, 2015) in PLATON (Spek, 2009), yielding a solvent accessible volume of $1316 \AA^{3}$ and 138 electrons. $\mathrm{H}$ atoms were fixed geometrically and refined using a riding model with $U(\mathrm{H})=$ $1.20 U_{\mathrm{eq}}(\mathrm{C})$.

\section{Acknowledgements}

This work was financially supported by the Otto-von-Guericke-Universität Magdeburg. SW holds a PhD studentship from the China Scholarship Council (CSC). FMS is grateful to the Ministry of Higher Educational Scientific Research (MHESR), Egypt, and the Germany Academic Exchange Service (DAAD), Germany, for a PhD scholarship within the German Egyptian Research Long-Term Scholarship (GERLS) program.

\section{References}

Brandenburg, K. (1999). DIAMOND. University of Bonn, Germany. Collins, S. (2011). Coord. Chem. Rev. 255, 118-138.

Deacon, G. B., Junk, P. C., Wang, J. \& Werner, D. (2014). Inorg. Chem. 53, 12553-12563.

Devi, A. (2013). Coord. Chem. Rev. 257, 3332-3384.

Dröse, P., Blaurock, S., Hrib, C. G., Hilfert, L. \& Edelmann, F. T. (2011). Z. Anorg. Allg. Chem. 637, 186-189.

Dröse, P., Hrib, C. G., Blaurock, S. \& Edelmann, F. T. (2010a). Acta Cryst. E66, m1474.

Dröse, P., Hrib, C. G. \& Edelmann, F. T. (2010b). J. Organomet. Chem. 695, 1953-1956.

Edelmann, F. T. (2009). Chem. Soc. Rev. 38, 2253-2268.

Edelmann, F. T. (2012). Chem. Soc. Rev. 41, 7657-7672.

Edelmann, F. T. (2013). Adv. Organomet. Chem. 61, 55-374.

Freeman, J. H. \& Smith, M. L. (1958). J. Inorg. Nucl. Chem. 7, $224-$ 227.

Kühling, M., Wickleder, C., Ferguson, M. J., Hrib, C. G., McDonald, R., Suta, M., Hilfert, L., Takats, J. \& Edelmann, F. T. (2015). New J. Chem. 39, 7617-7625.

Lamberts, W., Lueken, H. \& Hessner, B. (1987). Inorg. Chim. Acta, 134, 155-157.

Lueken, H., Lamberts, W. \& Hannibal, P. (1987). Inorg. Chim. Acta, 132, 111-118. 
Lueken, H., Schmitz, J., Lamberts, W., Hannibal, P. \& Handrick, K. (1989). Inorg. Chim. Acta, 156, 119-124.

Maginn, R. E., Manastyrskyj, S. \& Dubeck, M. (1963). J. Am. Chem. Soc. 85, 672-676.

Ong, T.-G., O'Brien, J. S., Korobkov, I. \& Richeson, D. S. (2006). Organometallics, 25, 4728-4730.

Richter, J., Feiling, J., Schmidt, H.-G., Noltemeyer, M., Brüser, W. \& Edelmann, F. T. (2004). Z. Anorg. Allg. Chem. 630, 1269-1275.

Rowley, C. N., DiLabio, G. A. \& Barry, S. T. (2005). Inorg. Chem. 44, 1983-1991.

Schumann, H., Keitsch, M. R., Winterfeld, J., Mühle, S. \& Molander, G. A. (1998). J. Organomet. Chem. 559, 181-190.

Seidel, W. W., Dachtler, W. \& Pape, T. (2012). Z. Anorg. Allg. Chem. 638, 116-121.

Sheldrick, G. M. (2008). Acta Cryst. A64, 112-122.

Sheldrick, G. M. (2015). Acta Cryst. C71, 3-8.

Sienkiewicz, P., Bielawski, K., Bielawska, A. \& Pałka, J. (2005). Environ. Toxicol. Pharmacol. 20, 118-124.

Spek, A. L. (2009). Acta Cryst. D65, 148-155.
Spek, A. L. (2015). Acta Cryst. C71, 9-18.

Sroor, F. M., Hrib, C. G., Hilfert, L., Busse, S. \& Edelmann, F. T. (2015a). New J. Chem. 39, 7595-7601.

Sroor, F. M., Hrib, C. G., Hilfert, L. \& Edelmann, F. T. (2013). Z. Anorg. Allg. Chem. 639, 2390-2394.

Sroor, F. M., Hrib, C. G., Hilfert, L., Hartenstein, L., Roesky, P. W. \& Edelmann, F. T. (2015b). J. Organomet. Chem. 799-800, 160-165.

Sroor, F. M., Hrib, C. G., Hilfert, L., Jones, P. G. \& Edelmann, F. T. (2015c). J. Organomet. Chem. 785, 1-10.

Sroor, F. M., Hrib, C. G., Liebing, P., Hilfert, L., Busse, S. \& Edelmann, F. T. (2016). Dalton Trans. doi: 10.1039/C6DT01974A.

Stoe \& Cie (2002). $X$-AREA and $X$-RED. Stoe \& Cie, Darmstadt, Germany.

Weingärtner, W. \& Maas, G. (2012). Eur. J. Org. Chem. pp. 63726382.

Xu, X., Gao, J., Cheng, D., Li, J., Qiang, G. \& Guo, H. (2008). Adv. Synth. Catal. 350, 61-64.

Xu, L., Wang, Y.-C., Zhang, W.-X. \& Xi, Z. (2013). Dalton Trans. 42, 16466-16469. 


\section{supporting information}

Acta Cryst. (2016). E72, 1229-1233 [https://doi.org/10.1107/S2056989016012135]

Crystal structures of two ytterbium(III) complexes comprising alkynylamidinate ligands

Sida Wang, Farid M. Sroor, Phil Liebing, Volker Lorenz, Liane Hilfert and Frank T. Edelmann

Computing details

For both compounds, data collection: $X$-AREA (Stoe \& Cie, 2002); cell refinement: $X$-AREA (Stoe \& Cie, 2002); data reduction: $X$-AREA and X-RED (Stoe \& Cie, 2002); program(s) used to solve structure: SHELXS2013 (Sheldrick, 2008); program(s) used to refine structure: SHELXL2014 (Sheldrick, 2015); molecular graphics: DIAMOND (Brandenburg, 1999); software used to prepare material for publication: SHELXL2014 (Sheldrick, 2015).

(li0090) Bis $\left(\eta^{5}\right.$-cyclopentadienyl) (3-cyclopropyl- $N, N^{\prime}$-diisopropylpropynamidinato- $\left.\kappa^{2} N, N^{\prime}\right)$ ytterbium(III)

Crystal data

$\left[\mathrm{Yb}\left(\mathrm{C}_{5} \mathrm{H}_{5}\right)_{2}\left(\mathrm{C}_{12} \mathrm{H}_{19} \mathrm{~N}_{2}\right)\right]$

$M_{r}=494.51$

Orthorhombic, $\mathrm{Pbca}$

$a=9.4578(2) \AA$

$b=19.2910(6) \AA$

$c=22.2114(5) \AA$

$V=4052.48(18) \AA^{3}$

$Z=8$

$F(000)=1960$

Data collection

Stoe IPDS 2T

diffractometer

Radiation source: fine-focus sealed tube

Detector resolution: 6.67 pixels $\mathrm{mm}^{-1}$

area detector scans

Absorption correction: numerical

(X-AREA and X-RED; Stoe \& Cie, 2002)

$T_{\min }=0.326, T_{\max }=0.448$

Refinement

Refinement on $F^{2}$

Least-squares matrix: full

$R\left[F^{2}>2 \sigma\left(F^{2}\right)\right]=0.018$

$w R\left(F^{2}\right)=0.040$

$S=0.98$

4045 reflections

227 parameters

0 restraints

Primary atom site location: heavy-atom method
$D_{\mathrm{x}}=1.621 \mathrm{Mg} \mathrm{m}^{-3}$

Mo $K \alpha$ radiation, $\lambda=0.71073 \AA$

Cell parameters from 21907 reflections

$\theta=1.8-26.2^{\circ}$

$\mu=4.62 \mathrm{~mm}^{-1}$

$T=153 \mathrm{~K}$

Block, orange

$0.33 \times 0.31 \times 0.25 \mathrm{~mm}$

21905 measured reflections

4045 independent reflections

3263 reflections with $I>2 \sigma(I)$

$R_{\text {int }}=0.051$

$\theta_{\text {max }}=26.2^{\circ}, \theta_{\min }=2.3^{\circ}$

$h=-11 \rightarrow 10$

$k=-23 \rightarrow 22$

$l=-27 \rightarrow 27$

Secondary atom site location: difference Fourier map

Hydrogen site location: inferred from neighbouring sites

$\mathrm{H}$-atom parameters constrained

$w=1 /\left[\sigma^{2}\left(F_{\mathrm{o}}^{2}\right)+(0.0189 P)^{2}\right]$

where $P=\left(F_{\mathrm{o}}^{2}+2 F_{\mathrm{c}}^{2}\right) / 3$

$(\Delta / \sigma)_{\max }<0.001$

$\Delta \rho_{\max }=0.50 \mathrm{e} \AA^{-3}$

$\Delta \rho_{\min }=-0.72$ e $\AA^{-3}$ 
Extinction correction: SHELXL2014 (Sheldrick, 2015),

$\mathrm{Fc}^{*}=\mathrm{kFc}\left[1+0.001 \times \mathrm{Fc}^{2} \lambda^{3} / \sin (2 \theta)\right]^{-1 / 4}$

Extinction coefficient: $0.00042(3)$

\section{Special details}

Geometry. All esds (except the esd in the dihedral angle between two 1.s. planes) are estimated using the full covariance matrix. The cell esds are taken into account individually in the estimation of esds in distances, angles and torsion angles; correlations between esds in cell parameters are only used when they are defined by crystal symmetry. An approximate (isotropic) treatment of cell esds is used for estimating esds involving l.s. planes.

Fractional atomic coordinates and isotropic or equivalent isotropic displacement parameters $\left(\AA^{2}\right)$

\begin{tabular}{|c|c|c|c|c|}
\hline & $x$ & $y$ & $z$ & $U_{\text {iso }} * / U_{\text {eq }}$ \\
\hline $\mathrm{C} 1$ & $0.3923(3)$ & $0.02660(12)$ & $0.12656(11)$ & $0.0209(5)$ \\
\hline $\mathrm{C} 2$ & $0.4476(3)$ & $-0.03844(14)$ & $0.14935(12)$ & $0.0243(5)$ \\
\hline $\mathrm{C} 3$ & $0.5016(3)$ & $-0.08748(13)$ & $0.17234(12)$ & $0.0237(5)$ \\
\hline $\mathrm{C} 4$ & $0.5728(3)$ & $-0.14441(13)$ & $0.20074(11)$ & $0.0239(5)$ \\
\hline $\mathrm{H} 1$ & 0.5401 & -0.1564 & 0.2423 & $0.029 *$ \\
\hline $\mathrm{C} 5$ & $0.7285(3)$ & $-0.15415(18)$ & $0.18859(17)$ & $0.0451(9)$ \\
\hline H3 & 0.7748 & -0.1214 & 0.1605 & $0.054^{*}$ \\
\hline $\mathrm{H} 2$ & 0.7888 & -0.1699 & 0.2224 & $0.054^{*}$ \\
\hline C6 & $0.6272(4)$ & $-0.20392(15)$ & $0.16337(14)$ & $0.0363(7)$ \\
\hline $\mathrm{H} 4$ & 0.6240 & -0.2509 & 0.1813 & $0.044^{*}$ \\
\hline H5 & 0.6100 & -0.2024 & 0.1194 & $0.044^{*}$ \\
\hline $\mathrm{C} 7$ & $0.1682(3)$ & $0.00848(14)$ & $0.17826(13)$ & $0.0279(6)$ \\
\hline H6 & 0.2233 & -0.0186 & 0.2086 & $0.033^{*}$ \\
\hline $\mathrm{C} 8$ & $0.0678(3)$ & $0.05675(15)$ & $0.21099(14)$ & $0.0354(7)$ \\
\hline H9 & 0.0013 & 0.0294 & 0.2351 & $0.042 *$ \\
\hline H7 & 0.1218 & 0.0876 & 0.2375 & $0.042 *$ \\
\hline $\mathrm{H} 8$ & 0.0153 & 0.0845 & 0.1816 & $0.042 *$ \\
\hline $\mathrm{C} 9$ & $0.0881(3)$ & $-0.04153(15)$ & $0.13814(15)$ & $0.0396(7)$ \\
\hline H11 & 0.0232 & -0.0693 & 0.1627 & $0.048^{*}$ \\
\hline $\mathrm{H} 10$ & 0.0341 & -0.0154 & 0.1081 & $0.048^{*}$ \\
\hline H12 & 0.1553 & -0.0722 & 0.1177 & $0.048^{*}$ \\
\hline $\mathrm{C} 10$ & $0.6091(3)$ & $0.04894(13)$ & $0.07174(12)$ & $0.0265(6)$ \\
\hline H13 & 0.6102 & -0.0018 & 0.0627 & $0.032 *$ \\
\hline C11 & $0.7191(3)$ & $0.06331(19)$ & $0.12009(15)$ & $0.0407(8)$ \\
\hline H15 & 0.8127 & 0.0496 & 0.1054 & $0.049^{*}$ \\
\hline H16 & 0.7193 & 0.1129 & 0.1297 & $0.049^{*}$ \\
\hline H14 & 0.6961 & 0.0367 & 0.1564 & $0.049^{*}$ \\
\hline $\mathrm{C} 12$ & $0.6454(3)$ & $0.08777(16)$ & 0.01454 (14) & $0.0361(7)$ \\
\hline H1 8 & 0.7412 & 0.0753 & 0.0017 & $0.043^{*}$ \\
\hline H17 & 0.5779 & 0.0753 & -0.0171 & $0.043^{*}$ \\
\hline H19 & 0.6406 & 0.1378 & 0.0221 & $0.043 *$ \\
\hline $\mathrm{C} 13$ & $0.3678(4)$ & $0.19289(17)$ & $0.20356(15)$ & $0.0468(9)$ \\
\hline $\mathrm{H} 20$ & 0.3727 & 0.1576 & 0.2333 & $0.056^{*}$ \\
\hline $\mathrm{C} 14$ & $0.4768(4)$ & $0.21487(18)$ & $0.16796(18)$ & $0.0513(10)$ \\
\hline $\mathrm{H} 21$ & 0.5707 & 0.1974 & 0.1690 & $0.062 *$ \\
\hline
\end{tabular}




$\begin{array}{lllll}\mathrm{C} 15 & 0.4272(4) & 0.26772(16) & 0.12948(17) & 0.0458(9) \\ \mathrm{H} 22 & 0.4803 & 0.2920 & 0.0999 & 0.055^{*} \\ \mathrm{C} 16 & 0.2837(3) & 0.27732(14) & 0.14360(13) & 0.0333(7) \\ \mathrm{H} 23 & 0.2215 & 0.3098 & 0.1254 & 0.040^{*} \\ \mathrm{C} 17 & 0.2495(4) & 0.23090(15) & 0.18876(14) & 0.0368(7) \\ \mathrm{H} 24 & 0.1588 & 0.2260 & 0.2067 & 0.044^{*} \\ \mathrm{C} 18 & 0.2309(3) & 0.20903(15) & -0.00641(12) & 0.0292(6) \\ \mathrm{H} 25 & 0.2880 & 0.2466 & -0.0196 & 0.035^{*} \\ \mathrm{C} 19 & 0.1094(3) & 0.21427(15) & 0.02902(12) & 0.0308(6) \\ \mathrm{H} 26 & 0.0686 & 0.2558 & 0.0441 & 0.037^{*} \\ \mathrm{C} 20 & 0.0589(3) & 0.14683(19) & 0.03826(14) & 0.0456(9) \\ \mathrm{H} 27 & -0.0230 & 0.1346 & 0.0606 & 0.055^{*} \\ \mathrm{C} 21 & 0.1492(4) & 0.10076(17) & 0.00905(15) & 0.0470(9) \\ \mathrm{H} 28 & 0.1402 & 0.0517 & 0.0086 & 0.056^{*} \\ \mathrm{C} 22 & 0.2543(4) & 0.13851(15) & -0.01913(13) & 0.0368(7) \\ \mathrm{H} 29 & 0.3290 & 0.1201 & -0.0429 & 0.044^{*} \\ \mathrm{~N} 1 & 0.2658(2) & 0.05054(11) & 0.14281(10) & 0.0212(4) \\ \mathrm{N} 2 & 0.4664(2) & 0.06799(10) & 0.09026(10) & 0.0216(4) \\ \mathrm{Yb} & 0.29970(2) & 0.15407(2) & 0.09600(2) & 0.01886(5)\end{array}$

Atomic displacement parameters $\left(\AA^{2}\right)$

\begin{tabular}{lllllll}
\hline & $U^{11}$ & $U^{22}$ & $U^{33}$ & $U^{12}$ & $U^{13}$ & $U^{23}$ \\
\hline $\mathrm{C} 1$ & $0.0188(12)$ & $0.0168(11)$ & $0.0271(13)$ & $-0.0009(10)$ & $-0.0038(11)$ & $-0.0017(9)$ \\
$\mathrm{C} 2$ & $0.0197(13)$ & $0.0225(12)$ & $0.0308(13)$ & $0.0006(11)$ & $0.0012(10)$ & $-0.0002(11)$ \\
$\mathrm{C} 3$ & $0.0191(13)$ & $0.0243(13)$ & $0.0276(14)$ & $-0.0013(12)$ & $0.0011(11)$ & $-0.0005(10)$ \\
$\mathrm{C} 4$ & $0.0233(13)$ & $0.0235(13)$ & $0.0248(13)$ & $0.0024(11)$ & $0.0008(10)$ & $0.0049(10)$ \\
$\mathrm{C} 5$ & $0.0207(16)$ & $0.0513(19)$ & $0.063(2)$ & $0.0067(15)$ & $-0.0007(14)$ & $0.0297(17)$ \\
$\mathrm{C} 6$ & $0.044(2)$ & $0.0315(15)$ & $0.0335(16)$ & $0.0146(15)$ & $0.0064(14)$ & $0.0086(12)$ \\
$\mathrm{C} 7$ & $0.0194(14)$ & $0.0286(14)$ & $0.0356(15)$ & $0.0005(11)$ & $0.0026(11)$ & $0.0138(12)$ \\
$\mathrm{C} 8$ & $0.0289(15)$ & $0.0425(17)$ & $0.0347(15)$ & $0.0003(13)$ & $0.0108(13)$ & $0.0069(13)$ \\
$\mathrm{C} 9$ & $0.0248(15)$ & $0.0255(14)$ & $0.069(2)$ & $-0.0048(14)$ & $0.0090(15)$ & $0.0011(14)$ \\
$\mathrm{C} 10$ & $0.0189(13)$ & $0.0207(13)$ & $0.0400(14)$ & $0.0017(11)$ & $0.0080(12)$ & $-0.0030(11)$ \\
$\mathrm{C} 11$ & $0.0202(16)$ & $0.057(2)$ & $0.0453(18)$ & $0.0026(14)$ & $0.0030(13)$ & $0.0037(15)$ \\
$\mathrm{C} 12$ & $0.0281(15)$ & $0.0424(17)$ & $0.0377(17)$ & $-0.0037(14)$ & $0.0093(12)$ & $-0.0038(13)$ \\
$\mathrm{C} 13$ & $0.065(2)$ & $0.0368(17)$ & $0.0390(18)$ & $0.0037(18)$ & $-0.0226(18)$ & $-0.0134(14)$ \\
$\mathrm{C} 14$ & $0.0307(18)$ & $0.0423(19)$ & $0.081(3)$ & $0.0127(16)$ & $-0.0246(18)$ & $-0.0393(19)$ \\
$\mathrm{C} 15$ & $0.047(2)$ & $0.0275(15)$ & $0.063(2)$ & $-0.0175(16)$ & $0.0116(17)$ & $-0.0182(15)$ \\
$\mathrm{C} 16$ & $0.0367(18)$ & $0.0198(12)$ & $0.0434(16)$ & $0.0058(13)$ & $-0.0073(14)$ & $-0.0059(11)$ \\
$\mathrm{C} 17$ & $0.0409(17)$ & $0.0318(15)$ & $0.0377(16)$ & $-0.0032(15)$ & $0.0032(14)$ & $-0.0113(12)$ \\
$\mathrm{C} 18$ & $0.0290(17)$ & $0.0302(14)$ & $0.0284(14)$ & $0.0040(12)$ & $-0.0004(12)$ & $0.0090(11)$ \\
$\mathrm{C} 19$ & $0.0239(14)$ & $0.0404(16)$ & $0.0281(14)$ & $0.0095(13)$ & $-0.0019(12)$ & $0.0060(12)$ \\
$\mathrm{C} 20$ & $0.0282(16)$ & $0.068(2)$ & $0.0405(18)$ & $-0.0194(17)$ & $-0.0139(13)$ & $0.0210(17)$ \\
$\mathrm{C} 21$ & $0.069(3)$ & $0.0321(16)$ & $0.0396(19)$ & $-0.0118(18)$ & $-0.0301(17)$ & $0.0043(13)$ \\
$\mathrm{C} 22$ & $0.0532(19)$ & $0.0357(16)$ & $0.0216(14)$ & $0.0131(14)$ & $-0.0055(13)$ & $0.0006(11)$ \\
$\mathrm{N} 1$ & $0.0165(11)$ & $0.0190(10)$ & $0.0281(11)$ & $0.0019(9)$ & $0.0014(9)$ & $0.0040(8)$ \\
$\mathrm{N} 2$ & $0.0169(11)$ & $0.0182(10)$ & $0.0298(12)$ & $0.0014(8)$ & $0.0024(9)$ & $-0.0018(9)$ \\
$\mathrm{Yb}$ & $0.01845(6)$ & $0.01490(6)$ & $0.02324(6)$ & $0.00096(4)$ & $-0.00219(4)$ & $0.00011(4)$ \\
& & & & & &
\end{tabular}


Geometric parameters $\left(\AA,{ }^{\circ}\right)$

\begin{tabular}{|c|c|c|c|}
\hline $\mathrm{C} 1-\mathrm{N} 1$ & $1.332(3)$ & $\mathrm{C} 12-\mathrm{H} 19$ & 0.9800 \\
\hline $\mathrm{C} 1-\mathrm{N} 2$ & $1.334(3)$ & $\mathrm{C} 13-\mathrm{C} 14$ & $1.367(6)$ \\
\hline $\mathrm{C} 1-\mathrm{C} 2$ & $1.451(4)$ & $\mathrm{C} 13-\mathrm{C} 17$ & $1.378(5)$ \\
\hline $\mathrm{C} 1-\mathrm{Yb}$ & $2.697(2)$ & $\mathrm{C} 13-\mathrm{Yb}$ & $2.585(3)$ \\
\hline $\mathrm{C} 2-\mathrm{C} 3$ & $1.190(4)$ & $\mathrm{C} 13-\mathrm{H} 20$ & 0.9500 \\
\hline $\mathrm{C} 3-\mathrm{C} 4$ & $1.434(4)$ & $\mathrm{C} 14-\mathrm{C} 15$ & $1.411(5)$ \\
\hline $\mathrm{C} 4-\mathrm{C} 6$ & $1.507(4)$ & $\mathrm{C} 14-\mathrm{Yb}$ & $2.595(3)$ \\
\hline $\mathrm{C} 4-\mathrm{C} 5$ & $1.509(4)$ & $\mathrm{C} 14-\mathrm{H} 21$ & 0.9500 \\
\hline $\mathrm{C} 4-\mathrm{H} 1$ & 1.0000 & $\mathrm{C} 15-\mathrm{C} 16$ & $1.405(5)$ \\
\hline $\mathrm{C} 5-\mathrm{C} 6$ & $1.468(5)$ & $\mathrm{C} 15-\mathrm{Yb}$ & $2.610(3)$ \\
\hline $\mathrm{C} 5-\mathrm{H} 3$ & 0.9900 & $\mathrm{C} 15-\mathrm{H} 22$ & 0.9500 \\
\hline $\mathrm{C} 5-\mathrm{H} 2$ & 0.9900 & $\mathrm{C} 16-\mathrm{C} 17$ & $1.383(4)$ \\
\hline $\mathrm{C} 6-\mathrm{H} 4$ & 0.9900 & $\mathrm{C} 16-\mathrm{Yb}$ & $2.607(3)$ \\
\hline $\mathrm{C} 6-\mathrm{H} 5$ & 0.9900 & $\mathrm{C} 16-\mathrm{H} 23$ & 0.9500 \\
\hline $\mathrm{C} 7-\mathrm{N} 1$ & $1.460(3)$ & $\mathrm{C} 17-\mathrm{Yb}$ & $2.582(3)$ \\
\hline $\mathrm{C} 7-\mathrm{C} 9$ & $1.516(4)$ & $\mathrm{C} 17-\mathrm{H} 24$ & 0.9500 \\
\hline $\mathrm{C} 7-\mathrm{C} 8$ & $1.516(4)$ & $\mathrm{C} 18-\mathrm{C} 19$ & $1.396(4)$ \\
\hline $\mathrm{C} 7-\mathrm{H} 6$ & 1.0000 & $\mathrm{C} 18-\mathrm{C} 22$ & $1.407(4)$ \\
\hline $\mathrm{C} 8-\mathrm{H} 9$ & 0.9800 & $\mathrm{C} 18-\mathrm{Yb}$ & $2.592(3)$ \\
\hline $\mathrm{C} 8-\mathrm{H} 7$ & 0.9800 & $\mathrm{C} 18-\mathrm{H} 25$ & 0.9500 \\
\hline $\mathrm{C} 8-\mathrm{H} 8$ & 0.9800 & $\mathrm{C} 19-\mathrm{C} 20$ & $1.401(4)$ \\
\hline $\mathrm{C} 9-\mathrm{H} 11$ & 0.9800 & $\mathrm{C} 19-\mathrm{Yb}$ & $2.608(3)$ \\
\hline C9- $\mathrm{H} 10$ & 0.9800 & $\mathrm{C} 19-\mathrm{H} 26$ & 0.9500 \\
\hline $\mathrm{C} 9-\mathrm{H} 12$ & 0.9800 & $\mathrm{C} 20-\mathrm{C} 21$ & $1.393(5)$ \\
\hline $\mathrm{C} 10-\mathrm{N} 2$ & $1.458(3)$ & $\mathrm{C} 20-\mathrm{Yb}$ & $2.617(3)$ \\
\hline $\mathrm{C} 10-\mathrm{C} 12$ & $1.514(4)$ & $\mathrm{C} 20-\mathrm{H} 27$ & 0.9500 \\
\hline $\mathrm{C} 10-\mathrm{C} 11$ & $1.521(4)$ & $\mathrm{C} 21-\mathrm{C} 22$ & $1.382(5)$ \\
\hline $\mathrm{C} 10-\mathrm{H} 13$ & 1.0000 & $\mathrm{C} 21-\mathrm{Yb}$ & $2.610(3)$ \\
\hline C11-H15 & 0.9800 & $\mathrm{C} 21-\mathrm{H} 28$ & 0.9500 \\
\hline C11-H16 & 0.9800 & $\mathrm{C} 22-\mathrm{Yb}$ & $2.610(3)$ \\
\hline C11-H14 & 0.9800 & $\mathrm{C} 22-\mathrm{H} 29$ & 0.9500 \\
\hline $\mathrm{C} 12-\mathrm{H} 18$ & 0.9800 & $\mathrm{~N} 1-\mathrm{Yb}$ & $2.274(2)$ \\
\hline $\mathrm{C} 12-\mathrm{H} 17$ & 0.9800 & $\mathrm{~N} 2-\mathrm{Yb}$ & $2.293(2)$ \\
\hline $\mathrm{N} 1-\mathrm{C} 1-\mathrm{N} 2$ & $115.4(2)$ & $\mathrm{Yb}-\mathrm{C} 18-\mathrm{H} 25$ & 116.2 \\
\hline $\mathrm{N} 1-\mathrm{C} 1-\mathrm{C} 2$ & $122.0(2)$ & $\mathrm{C} 18-\mathrm{C} 19-\mathrm{C} 20$ & $107.2(3)$ \\
\hline $\mathrm{N} 2-\mathrm{C} 1-\mathrm{C} 2$ & $122.6(2)$ & $\mathrm{C} 18-\mathrm{C} 19-\mathrm{Yb}$ & $73.82(16)$ \\
\hline $\mathrm{N} 1-\mathrm{C} 1-\mathrm{Yb}$ & $57.36(12)$ & $\mathrm{C} 20-\mathrm{C} 19-\mathrm{Yb}$ & $74.83(16)$ \\
\hline $\mathrm{N} 2-\mathrm{C} 1-\mathrm{Yb}$ & $58.18(12)$ & $\mathrm{C} 18-\mathrm{C} 19-\mathrm{H} 26$ & 126.4 \\
\hline $\mathrm{C} 2-\mathrm{C} 1-\mathrm{Yb}$ & $173.46(18)$ & $\mathrm{C} 20-\mathrm{C} 19-\mathrm{H} 26$ & 126.4 \\
\hline $\mathrm{C} 3-\mathrm{C} 2-\mathrm{C} 1$ & $172.8(3)$ & $\mathrm{Yb}-\mathrm{C} 19-\mathrm{H} 26$ & 117.1 \\
\hline $\mathrm{C} 2-\mathrm{C} 3-\mathrm{C} 4$ & $177.1(3)$ & $\mathrm{C} 21-\mathrm{C} 20-\mathrm{C} 19$ & $108.4(3)$ \\
\hline $\mathrm{C} 3-\mathrm{C} 4-\mathrm{C} 6$ & $120.1(2)$ & $\mathrm{C} 21-\mathrm{C} 20-\mathrm{Yb}$ & $74.25(18)$ \\
\hline $\mathrm{C} 3-\mathrm{C} 4-\mathrm{C} 5$ & $118.3(2)$ & $\mathrm{C} 19-\mathrm{C} 20-\mathrm{Yb}$ & $74.06(16)$ \\
\hline $\mathrm{C} 6-\mathrm{C} 4-\mathrm{C} 5$ & $58.2(2)$ & $\mathrm{C} 21-\mathrm{C} 20-\mathrm{H} 27$ & 125.8 \\
\hline $\mathrm{C} 3-\mathrm{C} 4-\mathrm{H} 1$ & 116.0 & $\mathrm{C} 19-\mathrm{C} 20-\mathrm{H} 27$ & 125.8 \\
\hline
\end{tabular}




\begin{tabular}{|c|c|c|c|}
\hline $\mathrm{C} 6-\mathrm{C} 4-\mathrm{H} 1$ & 116.0 & $\mathrm{Yb}-\mathrm{C} 20-\mathrm{H} 27$ & 117.8 \\
\hline $\mathrm{C} 5-\mathrm{C} 4-\mathrm{H} 1$ & 116.0 & $\mathrm{C} 22-\mathrm{C} 21-\mathrm{C} 20$ & $108.4(3)$ \\
\hline $\mathrm{C} 6-\mathrm{C} 5-\mathrm{C} 4$ & $60.8(2)$ & $\mathrm{C} 22-\mathrm{C} 21-\mathrm{Yb}$ & $74.66(18)$ \\
\hline $\mathrm{C} 6-\mathrm{C} 5-\mathrm{H} 3$ & 117.7 & $\mathrm{C} 20-\mathrm{C} 21-\mathrm{Yb}$ & 74.84 (18) \\
\hline $\mathrm{C} 4-\mathrm{C} 5-\mathrm{H} 3$ & 117.7 & $\mathrm{C} 22-\mathrm{C} 21-\mathrm{H} 28$ & 125.8 \\
\hline $\mathrm{C} 6-\mathrm{C} 5-\mathrm{H} 2$ & 117.7 & $\mathrm{C} 20-\mathrm{C} 21-\mathrm{H} 28$ & 125.8 \\
\hline $\mathrm{C} 4-\mathrm{C} 5-\mathrm{H} 2$ & 117.7 & $\mathrm{Yb}-\mathrm{C} 21-\mathrm{H} 28$ & 116.7 \\
\hline $\mathrm{H} 3-\mathrm{C} 5-\mathrm{H} 2$ & 114.8 & $\mathrm{C} 21-\mathrm{C} 22-\mathrm{C} 18$ & $107.8(3)$ \\
\hline $\mathrm{C} 5-\mathrm{C} 6-\mathrm{C} 4$ & $61.0(2)$ & $\mathrm{C} 21-\mathrm{C} 22-\mathrm{Yb}$ & 74.64 (18) \\
\hline $\mathrm{C} 5-\mathrm{C} 6-\mathrm{H} 4$ & 117.7 & $\mathrm{C} 18-\mathrm{C} 22-\mathrm{Yb}$ & $73.61(16)$ \\
\hline $\mathrm{C} 4-\mathrm{C} 6-\mathrm{H} 4$ & 117.7 & $\mathrm{C} 21-\mathrm{C} 22-\mathrm{H} 29$ & 126.1 \\
\hline $\mathrm{C} 5-\mathrm{C} 6-\mathrm{H} 5$ & 117.7 & $\mathrm{C} 18-\mathrm{C} 22-\mathrm{H} 29$ & 126.1 \\
\hline $\mathrm{C} 4-\mathrm{C} 6-\mathrm{H} 5$ & 117.7 & $\mathrm{Yb}-\mathrm{C} 22-\mathrm{H} 29$ & 117.7 \\
\hline $\mathrm{H} 4-\mathrm{C} 6-\mathrm{H} 5$ & 114.8 & $\mathrm{C} 1-\mathrm{N} 1-\mathrm{C} 7$ & $121.4(2)$ \\
\hline $\mathrm{N} 1-\mathrm{C} 7-\mathrm{C} 9$ & $110.6(2)$ & $\mathrm{C} 1-\mathrm{N} 1-\mathrm{Yb}$ & 93.09 (15) \\
\hline $\mathrm{N} 1-\mathrm{C} 7-\mathrm{C} 8$ & $108.3(2)$ & $\mathrm{C} 7-\mathrm{N} 1-\mathrm{Yb}$ & $145.49(16)$ \\
\hline $\mathrm{C} 9-\mathrm{C} 7-\mathrm{C} 8$ & $111.1(2)$ & $\mathrm{C} 1-\mathrm{N} 2-\mathrm{C} 10$ & $120.4(2)$ \\
\hline $\mathrm{N} 1-\mathrm{C} 7-\mathrm{H} 6$ & 108.9 & $\mathrm{C} 1-\mathrm{N} 2-\mathrm{Yb}$ & $92.20(15)$ \\
\hline $\mathrm{C} 9-\mathrm{C} 7-\mathrm{H} 6$ & 108.9 & $\mathrm{C} 10-\mathrm{N} 2-\mathrm{Yb}$ & $146.57(16)$ \\
\hline $\mathrm{C} 8-\mathrm{C} 7-\mathrm{H} 6$ & 108.9 & $\mathrm{~N} 1-\mathrm{Yb}-\mathrm{N} 2$ & $59.11(7)$ \\
\hline $\mathrm{C} 7-\mathrm{C} 8-\mathrm{H} 9$ & 109.5 & $\mathrm{~N} 1-\mathrm{Yb}-\mathrm{C} 17$ & $96.51(9)$ \\
\hline $\mathrm{C} 7-\mathrm{C} 8-\mathrm{H} 7$ & 109.5 & $\mathrm{~N} 2-\mathrm{Yb}-\mathrm{C} 17$ & $125.91(9)$ \\
\hline $\mathrm{H} 9-\mathrm{C} 8-\mathrm{H} 7$ & 109.5 & $\mathrm{~N} 1-\mathrm{Yb}-\mathrm{C} 13$ & $82.36(10)$ \\
\hline $\mathrm{C} 7-\mathrm{C} 8-\mathrm{H} 8$ & 109.5 & $\mathrm{~N} 2-\mathrm{Yb}-\mathrm{C} 13$ & $95.16(10)$ \\
\hline $\mathrm{H} 9-\mathrm{C} 8-\mathrm{H} 8$ & 109.5 & $\mathrm{C} 17-\mathrm{Yb}-\mathrm{C} 13$ & $30.92(11)$ \\
\hline $\mathrm{H} 7-\mathrm{C} 8-\mathrm{H} 8$ & 109.5 & $\mathrm{~N} 1-\mathrm{Yb}-\mathrm{C} 18$ & $136.46(9)$ \\
\hline $\mathrm{C} 7-\mathrm{C} 9-\mathrm{H} 11$ & 109.5 & $\mathrm{~N} 2-\mathrm{Yb}-\mathrm{C} 18$ & $114.82(8)$ \\
\hline $\mathrm{C} 7-\mathrm{C} 9-\mathrm{H} 10$ & 109.5 & $\mathrm{C} 17-\mathrm{Yb}-\mathrm{C} 18$ & $114.78(10)$ \\
\hline $\mathrm{H} 11-\mathrm{C} 9-\mathrm{H} 10$ & 109.5 & $\mathrm{C} 13-\mathrm{Yb}-\mathrm{C} 18$ & $139.01(10)$ \\
\hline C7-C9-H12 & 109.5 & $\mathrm{~N} 1-\mathrm{Yb}-\mathrm{C} 14$ & $101.91(11)$ \\
\hline $\mathrm{H} 11-\mathrm{C} 9-\mathrm{H} 12$ & 109.5 & $\mathrm{~N} 2-\mathrm{Yb}-\mathrm{C} 14$ & $85.28(9)$ \\
\hline $\mathrm{H} 10-\mathrm{C} 9-\mathrm{H} 12$ & 109.5 & $\mathrm{C} 17-\mathrm{Yb}-\mathrm{C} 14$ & $50.80(11)$ \\
\hline $\mathrm{N} 2-\mathrm{C} 10-\mathrm{C} 12$ & $108.8(2)$ & $\mathrm{C} 13-\mathrm{Yb}-\mathrm{C} 14$ & $30.60(12)$ \\
\hline $\mathrm{N} 2-\mathrm{C} 10-\mathrm{C} 11$ & $112.8(2)$ & $\mathrm{C} 18-\mathrm{Yb}-\mathrm{C} 14$ & $121.15(12)$ \\
\hline $\mathrm{C} 12-\mathrm{C} 10-\mathrm{C} 11$ & $110.3(2)$ & $\mathrm{N} 1-\mathrm{Yb}-\mathrm{C} 16$ & $127.40(9)$ \\
\hline $\mathrm{N} 2-\mathrm{C} 10-\mathrm{H} 13$ & 108.3 & $\mathrm{~N} 2-\mathrm{Yb}-\mathrm{C} 16$ & $136.29(9)$ \\
\hline $\mathrm{C} 12-\mathrm{C} 10-\mathrm{H} 13$ & 108.3 & $\mathrm{C} 17-\mathrm{Yb}-\mathrm{C} 16$ & $30.92(10)$ \\
\hline $\mathrm{C} 11-\mathrm{C} 10-\mathrm{H} 13$ & 108.3 & $\mathrm{C} 13-\mathrm{Yb}-\mathrm{C} 16$ & 51.34 (10) \\
\hline $\mathrm{C} 10-\mathrm{C} 11-\mathrm{H} 15$ & 109.5 & $\mathrm{C} 18-\mathrm{Yb}-\mathrm{C} 16$ & $88.18(9)$ \\
\hline $\mathrm{C} 10-\mathrm{C} 11-\mathrm{H} 16$ & 109.5 & $\mathrm{C} 14-\mathrm{Yb}-\mathrm{C} 16$ & $51.34(10)$ \\
\hline $\mathrm{H} 15-\mathrm{C} 11-\mathrm{H} 16$ & 109.5 & $\mathrm{~N} 1-\mathrm{Yb}-\mathrm{C} 19$ & $123.68(8)$ \\
\hline $\mathrm{C} 10-\mathrm{C} 11-\mathrm{H} 14$ & 109.5 & $\mathrm{~N} 2-\mathrm{Yb}-\mathrm{C} 19$ & $139.92(8)$ \\
\hline $\mathrm{H} 15-\mathrm{C} 11-\mathrm{H} 14$ & 109.5 & $\mathrm{C} 17-\mathrm{Yb}-\mathrm{C} 19$ & $94.16(10)$ \\
\hline $\mathrm{H} 16-\mathrm{C} 11-\mathrm{H} 14$ & 109.5 & $\mathrm{C} 13-\mathrm{Yb}-\mathrm{C} 19$ & $124.76(10)$ \\
\hline $\mathrm{C} 10-\mathrm{C} 12-\mathrm{H} 18$ & 109.5 & $\mathrm{C} 18-\mathrm{Yb}-\mathrm{C} 19$ & $31.15(9)$ \\
\hline $\mathrm{C} 10-\mathrm{C} 12-\mathrm{H} 17$ & 109.5 & $\mathrm{C} 14-\mathrm{Yb}-\mathrm{C} 19$ & $126.54(11)$ \\
\hline $\mathrm{H} 18-\mathrm{C} 12-\mathrm{H} 17$ & 109.5 & $\mathrm{C} 16-\mathrm{Yb}-\mathrm{C} 19$ & $77.60(9)$ \\
\hline $\mathrm{C} 10-\mathrm{C} 12-\mathrm{H} 19$ & 109.5 & $\mathrm{~N} 1-\mathrm{Yb}-\mathrm{C} 21$ & $85.14(9)$ \\
\hline
\end{tabular}




\begin{tabular}{|c|c|c|c|}
\hline $\mathrm{H} 18-\mathrm{C} 12-\mathrm{H} 19$ & 109.5 & $\mathrm{~N} 2-\mathrm{Yb}-\mathrm{C} 21$ & $92.78(10)$ \\
\hline $\mathrm{H} 17-\mathrm{C} 12-\mathrm{H} 19$ & 109.5 & $\mathrm{C} 17-\mathrm{Yb}-\mathrm{C} 21$ & $135.74(12)$ \\
\hline $\mathrm{C} 14-\mathrm{C} 13-\mathrm{C} 17$ & $108.0(3)$ & $\mathrm{C} 13-\mathrm{Yb}-\mathrm{C} 21$ & $159.02(13)$ \\
\hline $\mathrm{C} 14-\mathrm{C} 13-\mathrm{Yb}$ & $75.12(19)$ & $\mathrm{C} 18-\mathrm{Yb}-\mathrm{C} 21$ & $51.33(10)$ \\
\hline $\mathrm{C} 17-\mathrm{C} 13-\mathrm{Yb}$ & 74.42 (17) & $\mathrm{C} 14-\mathrm{Yb}-\mathrm{C} 21$ & $170.27(13)$ \\
\hline $\mathrm{C} 14-\mathrm{C} 13-\mathrm{H} 20$ & 126.0 & $\mathrm{C} 16-\mathrm{Yb}-\mathrm{C} 21$ & $128.90(10)$ \\
\hline $\mathrm{C} 17-\mathrm{C} 13-\mathrm{H} 20$ & 126.0 & $\mathrm{C} 19-\mathrm{Yb}-\mathrm{C} 21$ & $51.47(10)$ \\
\hline $\mathrm{Yb}-\mathrm{C} 13-\mathrm{H} 20$ & 116.6 & $\mathrm{~N} 1-\mathrm{Yb}-\mathrm{C} 15$ & $132.26(10)$ \\
\hline $\mathrm{C} 13-\mathrm{C} 14-\mathrm{C} 15$ & $108.9(3)$ & $\mathrm{N} 2-\mathrm{Yb}-\mathrm{C} 15$ & $107.84(10)$ \\
\hline $\mathrm{C} 13-\mathrm{C} 14-\mathrm{Yb}$ & 74.29 (19) & $\mathrm{C} 17-\mathrm{Yb}-\mathrm{C} 15$ & $51.36(10)$ \\
\hline $\mathrm{C} 15-\mathrm{C} 14-\mathrm{Yb}$ & 74.87 (18) & $\mathrm{C} 13-\mathrm{Yb}-\mathrm{C} 15$ & $51.56(12)$ \\
\hline $\mathrm{C} 13-\mathrm{C} 14-\mathrm{H} 21$ & 125.5 & $\mathrm{C} 18-\mathrm{Yb}-\mathrm{C} 15$ & $91.28(11)$ \\
\hline $\mathrm{C} 15-\mathrm{C} 14-\mathrm{H} 21$ & 125.5 & $\mathrm{C} 14-\mathrm{Yb}-\mathrm{C} 15$ & 31.44 (12) \\
\hline $\mathrm{Yb}-\mathrm{C} 14-\mathrm{H} 21$ & 117.2 & $\mathrm{C} 16-\mathrm{Yb}-\mathrm{C} 15$ & $31.25(10)$ \\
\hline $\mathrm{C} 16-\mathrm{C} 15-\mathrm{C} 14$ & $106.3(3)$ & $\mathrm{C} 19-\mathrm{Yb}-\mathrm{C} 15$ & $96.16(11)$ \\
\hline $\mathrm{C} 16-\mathrm{C} 15-\mathrm{Yb}$ & $74.22(17)$ & $\mathrm{C} 21-\mathrm{Yb}-\mathrm{C} 15$ & $142.52(11)$ \\
\hline $\mathrm{C} 14-\mathrm{C} 15-\mathrm{Yb}$ & 73.69 (17) & $\mathrm{N} 1-\mathrm{Yb}-\mathrm{C} 22$ & $108.89(9)$ \\
\hline $\mathrm{C} 16-\mathrm{C} 15-\mathrm{H} 22$ & 126.8 & $\mathrm{~N} 2-\mathrm{Yb}-\mathrm{C} 22$ & $88.59(9)$ \\
\hline $\mathrm{C} 14-\mathrm{C} 15-\mathrm{H} 22$ & 126.8 & $\mathrm{C} 17-\mathrm{Yb}-\mathrm{C} 22$ & $144.82(10)$ \\
\hline $\mathrm{Yb}-\mathrm{C} 15-\mathrm{H} 22$ & 117.4 & $\mathrm{C} 13-\mathrm{Yb}-\mathrm{C} 22$ & $168.41(11)$ \\
\hline $\mathrm{C} 17-\mathrm{C} 16-\mathrm{C} 15$ & $107.6(3)$ & $\mathrm{C} 18-\mathrm{Yb}-\mathrm{C} 22$ & $31.38(9)$ \\
\hline $\mathrm{C} 17-\mathrm{C} 16-\mathrm{Yb}$ & 73.57 (16) & $\mathrm{C} 14-\mathrm{Yb}-\mathrm{C} 22$ & $139.59(13)$ \\
\hline $\mathrm{C} 15-\mathrm{C} 16-\mathrm{Yb}$ & $74.53(16)$ & $\mathrm{C} 16-\mathrm{Yb}-\mathrm{C} 22$ & $119.52(9)$ \\
\hline $\mathrm{C} 17-\mathrm{C} 16-\mathrm{H} 23$ & 126.2 & $\mathrm{C} 19-\mathrm{Yb}-\mathrm{C} 22$ & $51.59(9)$ \\
\hline $\mathrm{C} 15-\mathrm{C} 16-\mathrm{H} 23$ & 126.2 & $\mathrm{C} 21-\mathrm{Yb}-\mathrm{C} 22$ & $30.70(11)$ \\
\hline $\mathrm{Yb}-\mathrm{C} 16-\mathrm{H} 23$ & 117.7 & $\mathrm{C} 15-\mathrm{Yb}-\mathrm{C} 22$ & $116.85(11)$ \\
\hline $\mathrm{C} 13-\mathrm{C} 17-\mathrm{C} 16$ & $109.1(3)$ & $\mathrm{N} 1-\mathrm{Yb}-\mathrm{C} 20$ & $93.12(9)$ \\
\hline $\mathrm{C} 13-\mathrm{C} 17-\mathrm{Yb}$ & $74.66(17)$ & $\mathrm{N} 2-\mathrm{Yb}-\mathrm{C} 20$ & $122.16(10)$ \\
\hline $\mathrm{C} 16-\mathrm{C} 17-\mathrm{Yb}$ & $75.52(17)$ & $\mathrm{C} 17-\mathrm{Yb}-\mathrm{C} 20$ & $105.16(12)$ \\
\hline $\mathrm{C} 13-\mathrm{C} 17-\mathrm{H} 24$ & 125.4 & $\mathrm{C} 13-\mathrm{Yb}-\mathrm{C} 20$ & $133.23(12)$ \\
\hline $\mathrm{C} 16-\mathrm{C} 17-\mathrm{H} 24$ & 125.4 & $\mathrm{C} 18-\mathrm{Yb}-\mathrm{C} 20$ & $51.22(9)$ \\
\hline $\mathrm{Yb}-\mathrm{C} 17-\mathrm{H} 24$ & 116.3 & $\mathrm{C} 14-\mathrm{Yb}-\mathrm{C} 20$ & $152.55(11)$ \\
\hline $\mathrm{C} 19-\mathrm{C} 18-\mathrm{C} 22$ & $108.2(3)$ & $\mathrm{C} 16-\mathrm{Yb}-\mathrm{C} 20$ & $101.36(11)$ \\
\hline $\mathrm{C} 19-\mathrm{C} 18-\mathrm{Yb}$ & $75.03(15)$ & $\mathrm{C} 19-\mathrm{Yb}-\mathrm{C} 20$ & $31.11(10)$ \\
\hline $\mathrm{C} 22-\mathrm{C} 18-\mathrm{Yb}$ & $75.01(16)$ & $\mathrm{C} 21-\mathrm{Yb}-\mathrm{C} 20$ & $30.91(11)$ \\
\hline $\mathrm{C} 19-\mathrm{C} 18-\mathrm{H} 25$ & 125.9 & $\mathrm{C} 15-\mathrm{Yb}-\mathrm{C} 20$ & $125.90(12)$ \\
\hline $\mathrm{C} 22-\mathrm{C} 18-\mathrm{H} 25$ & 125.9 & $\mathrm{C} 22-\mathrm{Yb}-\mathrm{C} 20$ & $51.00(11)$ \\
\hline $\mathrm{C} 3-\mathrm{C} 4-\mathrm{C} 5-\mathrm{C} 6$ & $-109.6(3)$ & $\mathrm{C} 19-\mathrm{C} 20-\mathrm{C} 21-\mathrm{Yb}$ & $66.8(2)$ \\
\hline $\mathrm{C} 3-\mathrm{C} 4-\mathrm{C} 6-\mathrm{C} 5$ & $106.6(3)$ & $\mathrm{C} 20-\mathrm{C} 21-\mathrm{C} 22-\mathrm{C} 18$ & $1.1(3)$ \\
\hline $\mathrm{C} 17-\mathrm{C} 13-\mathrm{C} 14-\mathrm{C} 15$ & $-0.3(3)$ & $\mathrm{Yb}-\mathrm{C} 21-\mathrm{C} 22-\mathrm{C} 18$ & $-66.7(2)$ \\
\hline $\mathrm{Yb}-\mathrm{C} 13-\mathrm{C} 14-\mathrm{C} 15$ & $67.5(2)$ & $\mathrm{C} 20-\mathrm{C} 21-\mathrm{C} 22-\mathrm{Yb}$ & $67.8(2)$ \\
\hline $\mathrm{C} 17-\mathrm{C} 13-\mathrm{C} 14-\mathrm{Yb}$ & $-67.7(2)$ & $\mathrm{C} 19-\mathrm{C} 18-\mathrm{C} 22-\mathrm{C} 21$ & $-0.9(3)$ \\
\hline $\mathrm{C} 13-\mathrm{C} 14-\mathrm{C} 15-\mathrm{C} 16$ & $0.5(3)$ & $\mathrm{Yb}-\mathrm{C} 18-\mathrm{C} 22-\mathrm{C} 21$ & $67.4(2)$ \\
\hline $\mathrm{Yb}-\mathrm{C} 14-\mathrm{C} 15-\mathrm{C} 16$ & $67.6(2)$ & $\mathrm{C} 19-\mathrm{C} 18-\mathrm{C} 22-\mathrm{Yb}$ & $-68.3(2)$ \\
\hline $\mathrm{C} 13-\mathrm{C} 14-\mathrm{C} 15-\mathrm{Yb}$ & $-67.1(2)$ & $\mathrm{N} 2-\mathrm{C} 1-\mathrm{N} 1-\mathrm{C} 7$ & $174.8(2)$ \\
\hline $\mathrm{C} 14-\mathrm{C} 15-\mathrm{C} 16-\mathrm{C} 17$ & $-0.6(3)$ & $\mathrm{C} 2-\mathrm{C} 1-\mathrm{N} 1-\mathrm{C} 7$ & $-8.1(4)$ \\
\hline $\mathrm{Yb}-\mathrm{C} 15-\mathrm{C} 16-\mathrm{C} 17$ & $66.7(2)$ & $\mathrm{Yb}-\mathrm{C} 1-\mathrm{N} 1-\mathrm{C} 7$ & $179.6(3)$ \\
\hline
\end{tabular}




$\begin{array}{llll}\mathrm{C} 14-\mathrm{C} 15-\mathrm{C} 16-\mathrm{Yb} & -67.2(2) & \mathrm{N} 2-\mathrm{C} 1-\mathrm{N} 1-\mathrm{Yb} & -4.8(2) \\ \mathrm{C} 14-\mathrm{C} 13-\mathrm{C} 17-\mathrm{C} 16 & -0.1(3) & \mathrm{C} 2-\mathrm{C} 1-\mathrm{N} 1-\mathrm{Yb} & 172.3(2) \\ \mathrm{Yb}-\mathrm{C} 13-\mathrm{C} 17-\mathrm{C} 16 & -68.3(2) & \mathrm{C} 9-\mathrm{C} 7-\mathrm{N} 1-\mathrm{C} 1 & -81.7(3) \\ \mathrm{C} 14-\mathrm{C} 13-\mathrm{C} 17-\mathrm{Yb} & 68.2(2) & \mathrm{C} 8-\mathrm{C} 7-\mathrm{N} 1-\mathrm{C} 1 & 156.3(2) \\ \mathrm{C} 15-\mathrm{C} 16-\mathrm{C} 17-\mathrm{C} 13 & 0.4(3) & \mathrm{C} 9-\mathrm{C} 7-\mathrm{N} 1-\mathrm{Yb} & 97.6(3) \\ \mathrm{Yb}-\mathrm{C} 16-\mathrm{C} 17-\mathrm{C} 13 & 67.7(2) & \mathrm{C} 8-\mathrm{C} 7-\mathrm{N} 1-\mathrm{Yb} & -24.4(4) \\ \mathrm{C} 15-\mathrm{C} 16-\mathrm{C} 17-\mathrm{Yb} & -67.3(2) & \mathrm{N} 1-\mathrm{C} 1-\mathrm{N} 2-\mathrm{C} 10 & 177.0(2) \\ \mathrm{C} 22-\mathrm{C} 18-\mathrm{C} 19-\mathrm{C} 20 & 0.3(3) & \mathrm{C} 2-\mathrm{C} 1-\mathrm{N} 2-\mathrm{C} 10 & 0.0(4) \\ \mathrm{Yb}-\mathrm{C} 18-\mathrm{C} 19-\mathrm{C} 20 & -68.0(2) & \mathrm{Yb}-\mathrm{C} 1-\mathrm{N} 2-\mathrm{C} 10 & 172.3(3) \\ \mathrm{C} 22-\mathrm{C} 18-\mathrm{C} 19-\mathrm{Yb} & 68.3(2) & \mathrm{N} 1-\mathrm{C} 1-\mathrm{N} 2-\mathrm{Yb} & 4.7(2) \\ \mathrm{C} 18-\mathrm{C} 19-\mathrm{C} 20-\mathrm{C} 21 & 0.4(3) & \mathrm{C} 2-\mathrm{C} 1-\mathrm{N} 2-\mathrm{Yb} & -172.3(2) \\ \mathrm{Yb}-\mathrm{C} 19-\mathrm{C} 20-\mathrm{C} 21 & -66.9(2) & \mathrm{C} 12-\mathrm{C} 10-\mathrm{N} 2-\mathrm{C} 1 & 158.5(2) \\ \mathrm{C} 18-\mathrm{C} 19-\mathrm{C} 20-\mathrm{Yb} & 67.3(2) & \mathrm{C} 11-\mathrm{C} 10-\mathrm{N} 2-\mathrm{C} 1 & -78.8(3) \\ \mathrm{C} 19-\mathrm{C} 20-\mathrm{C} 21-\mathrm{C} 22 & -0.9(4) & \mathrm{C} 12-\mathrm{C} 10-\mathrm{N} 2-\mathrm{Yb} & -35.6(4) \\ \mathrm{Yb}-\mathrm{C} 20-\mathrm{C} 21-\mathrm{C} 22 & -67.7(2) & \mathrm{C} 11-\mathrm{C} 10-\mathrm{N} 2-\mathrm{Yb} & 87.2(4)\end{array}$

(li0065_sq) Tris(3-phenyl- $N, N^{\prime}$-dicyclohexylpropynamidinato- $\left.\kappa^{2} N, N^{\prime}\right)$ ytterbium(III)

\section{Crystal data}

$\left[\mathrm{Yb}\left(\mathrm{C}_{21} \mathrm{H}_{27} \mathrm{~N}_{2}\right)_{3}\right]$

$M_{r}=1095.37$

Trigonal, $R \overline{3} c: H$

$a=20.3469(3) \AA$

$c=50.3074(11) \AA$

$V=18036.8(7) \AA^{3}$

$Z=12$

$F(000)=6852$

\section{Data collection}

Stoe IPDS 2T diffractometer

Radiation source: fine-focus sealed tube Detector resolution: 6.67 pixels $\mathrm{mm}^{-1}$ area detector scans

Absorption correction: numerical

(X-AREA and X-RED; Stoe \& Cie, 2002)

$T_{\min }=0.621, T_{\max }=0.722$

\section{Refinement}

Refinement on $F^{2}$

Least-squares matrix: full

$R\left[F^{2}>2 \sigma\left(F^{2}\right)\right]=0.029$

$w R\left(F^{2}\right)=0.071$

$S=1.07$

3578 reflections

257 parameters

0 restraints

Primary atom site location: heavy-atom method
$D_{\mathrm{x}}=1.210 \mathrm{Mg} \mathrm{m}^{-3}$

Mo $K \alpha$ radiation, $\lambda=0.71073 \AA$

Cell parameters from 36835 reflections

$\theta=2.0-25.1^{\circ}$

$\mu=1.60 \mathrm{~mm}^{-1}$

$T=153 \mathrm{~K}$

Block, light yellow

$0.36 \times 0.35 \times 0.24 \mathrm{~mm}$

36832 measured reflections

3578 independent reflections

2774 reflections with $I>2 \sigma(I)$

$R_{\text {int }}=0.065$

$\theta_{\max }=25.1^{\circ}, \theta_{\min }=2.0^{\circ}$

$h=-24 \rightarrow 24$

$k=-24 \rightarrow 24$

$l=-59 \rightarrow 59$

Secondary atom site location: difference Fourier map

Hydrogen site location: inferred from neighbouring sites

$\mathrm{H}$-atom parameters constrained

$w=1 /\left[\sigma^{2}\left(F_{\mathrm{o}}{ }^{2}\right)+(0.0274 P)^{2}+35.9511 P\right]$

where $P=\left(F_{\mathrm{o}}{ }^{2}+2 F_{\mathrm{c}}{ }^{2}\right) / 3$

$(\Delta / \sigma)_{\max }=0.001$

$\Delta \rho_{\max }=0.31 \mathrm{e}^{-3}$

$\Delta \rho_{\min }=-1.18$ e $\AA^{-3}$ 


\section{Special details}

Geometry. All esds (except the esd in the dihedral angle between two 1.s. planes) are estimated using the full covariance matrix. The cell esds are taken into account individually in the estimation of esds in distances, angles and torsion angles; correlations between esds in cell parameters are only used when they are defined by crystal symmetry. An approximate (isotropic) treatment of cell esds is used for estimating esds involving l.s. planes.

Refinement. PLATON SQUEEZE (Spek, 2015)

Fractional atomic coordinates and isotropic or equivalent isotropic displacement parameters $\left(\AA^{2}\right)$

\begin{tabular}{|c|c|c|c|c|c|}
\hline & $x$ & $y$ & $z$ & $U_{\text {iso }} * / U_{\text {eq }}$ & Occ. $(<1)$ \\
\hline $\mathrm{C} 1$ & $0.11736(16)$ & $0.97236(16)$ & $0.14621(6)$ & $0.0488(7)$ & \\
\hline $\mathrm{C} 2$ & $0.18462(17)$ & $0.96500(16)$ & $0.14454(7)$ & $0.0553(8)$ & \\
\hline $\mathrm{C} 3$ & $0.24269(17)$ & $0.96456(16)$ & $0.14323(7)$ & $0.0569(8)$ & \\
\hline $\mathrm{C} 4$ & $0.31432(16)$ & $0.96673(17)$ & $0.14298(7)$ & $0.0552(8)$ & \\
\hline $\mathrm{C} 5$ & $0.36272(19)$ & $0.9967(2)$ & $0.16454(8)$ & $0.0644(9)$ & \\
\hline H1 & 0.3477 & 1.0145 & 0.1795 & $0.077^{*}$ & \\
\hline C6 & $0.4328(2)$ & $1.0010(2)$ & $0.16442(9)$ & $0.0773(11)$ & \\
\hline $\mathrm{H} 2$ & 0.4658 & 1.0217 & 0.1792 & $0.093 *$ & \\
\hline $\mathrm{C} 7$ & $0.4543(2)$ & $0.9751(2)$ & $0.14279(10)$ & $0.0853(13)$ & \\
\hline H3 & 0.5017 & 0.9768 & 0.1429 & $0.102 *$ & \\
\hline $\mathrm{C} 8$ & $0.4074(2)$ & $0.9468(2)$ & $0.12089(10)$ & $0.0847(13)$ & \\
\hline $\mathrm{H} 4$ & 0.4233 & 0.9302 & 0.1058 & $0.102 *$ & \\
\hline C9 & $0.3374(2)$ & $0.9423(2)$ & $0.12084(9)$ & $0.0712(10)$ & \\
\hline H5 & 0.3052 & 0.9228 & 0.1058 & $0.085^{*}$ & \\
\hline $\mathrm{C} 10$ & $0.06136(18)$ & $0.8906(2)$ & $0.10821(7)$ & $0.0610(9)$ & \\
\hline H6 & 0.0980 & 0.8741 & 0.1138 & $0.073 *$ & \\
\hline $\mathrm{C} 11$ & $0.0855(2)$ & $0.9272(3)$ & $0.08192(8)$ & 0.0935 (14) & \\
\hline H8 & 0.1378 & 0.9707 & 0.0831 & $0.112 *$ & \\
\hline $\mathrm{H} 7$ & 0.0518 & 0.9468 & 0.0764 & $0.112^{*}$ & \\
\hline C12 & $0.0825(3)$ & 0.8709 & $0.06098(11)$ & $0.134(2)$ & \\
\hline $\mathrm{H} 10$ & 0.0972 & 0.8960 & 0.0434 & $0.161^{*}$ & \\
\hline H9 & 0.1190 & 0.8540 & 0.0657 & $0.161^{*}$ & \\
\hline $\mathrm{C} 13$ & $0.0034(3)$ & 0.8030 & $0.05952(11)$ & $0.131(2)$ & \\
\hline H12 & -0.0324 & 0.8195 & 0.0536 & $0.157^{*}$ & \\
\hline H11 & 0.0023 & 0.7663 & 0.0463 & $0.157^{*}$ & \\
\hline $\mathrm{C} 14$ & $-0.0206(2)$ & $0.7657(3)$ & $0.08578(11)$ & 0.1059 (18) & \\
\hline H13 & 0.0125 & 0.7450 & 0.0910 & $0.127^{*}$ & \\
\hline H14 & -0.0733 & 0.7227 & 0.0846 & $0.127^{*}$ & \\
\hline C15 & $-0.0164(2)$ & 0.82102 (19) & $0.10691(8)$ & $0.0691(10)$ & \\
\hline H15 & -0.0544 & 0.8365 & 0.1029 & $0.083 *$ & \\
\hline H16 & -0.0290 & 0.7955 & 0.1244 & $0.083 *$ & \\
\hline C16A & $0.17017(18)$ & $1.05213(17)$ & $0.18498(7)$ & $0.0566(8)$ & $0.442(4)$ \\
\hline H17A & 0.2173 & 1.0728 & 0.1739 & $0.068 *$ & $0.442(4)$ \\
\hline $\mathrm{C} 16 \mathrm{~B}$ & $0.17017(18)$ & $1.05213(17)$ & $0.18498(7)$ & $0.0566(8)$ & $0.558(4)$ \\
\hline H17B & 0.1932 & 1.0199 & 0.1891 & $0.068 *$ & $0.558(4)$ \\
\hline C17A & $0.1797(4)$ & $1.0071(4)$ & $0.20382(16)$ & $0.065(2)$ & $0.442(4)$ \\
\hline H18A & 0.1894 & 0.9698 & 0.1946 & $0.078^{*}$ & $0.442(4)$ \\
\hline H19A & 0.1324 & 0.9786 & 0.2142 & $0.078 *$ & $0.442(4)$ \\
\hline
\end{tabular}




\begin{tabular}{|c|c|c|c|c|c|}
\hline C17B & $0.1324(4)$ & $1.0618(3)$ & $0.21256(12)$ & 0.0644 (17) & $0.558(4)$ \\
\hline H18B & 0.1064 & 1.0909 & 0.2087 & $0.077 *$ & $0.558(4)$ \\
\hline H19B & 0.0938 & 1.0111 & 0.2191 & $0.077 *$ & $0.558(4)$ \\
\hline C18A & $0.2455(6)$ & $1.0543(5)$ & $0.2227(2)$ & $0.091(3)$ & $0.442(4)$ \\
\hline $\mathrm{H} 20 \mathrm{~A}$ & 0.2459 & 1.0217 & 0.2373 & $0.109 *$ & $0.442(4)$ \\
\hline $\mathrm{H} 21 \mathrm{~A}$ & 0.2942 & 1.0753 & 0.2130 & $0.109 *$ & $0.442(4)$ \\
\hline C18B & $0.1916(6)$ & $1.1025(6)$ & $0.23422(18)$ & $0.080(3)$ & $0.558(4)$ \\
\hline H20B & 0.1677 & 1.1124 & 0.2497 & $0.096^{*}$ & $0.558(4)$ \\
\hline $\mathrm{H} 21 \mathrm{~B}$ & 0.2121 & 1.0699 & 0.2402 & $0.096^{*}$ & $0.558(4)$ \\
\hline C19A & $0.2374(8)$ & $1.1184(9)$ & $0.2342(2)$ & $0.089(4)$ & $0.442(4)$ \\
\hline $\mathrm{H} 22 \mathrm{~A}$ & 0.2808 & 1.1492 & 0.2462 & $0.106^{*}$ & $0.442(4)$ \\
\hline $\mathrm{H} 23 \mathrm{~A}$ & 0.1904 & 1.0971 & 0.2450 & $0.106^{*}$ & $0.442(4)$ \\
\hline C19B & $0.2552(6)$ & $1.1765(6)$ & $0.2237(2)$ & $0.074(3)$ & $0.558(4)$ \\
\hline $\mathrm{H} 22 \mathrm{~B}$ & 0.2949 & 1.2007 & 0.2375 & $0.088^{*}$ & $0.558(4)$ \\
\hline $\mathrm{H} 23 \mathrm{~B}$ & 0.2354 & 1.2111 & 0.2198 & $0.088^{*}$ & $0.558(4)$ \\
\hline $\mathrm{C} 20 \mathrm{~A}$ & $0.2345(7)$ & $1.1693(8)$ & $0.2125(2)$ & $0.067(3)$ & $0.442(4)$ \\
\hline $\mathrm{H} 24 \mathrm{~A}$ & 0.2269 & 1.2094 & 0.2206 & $0.080 *$ & $0.442(4)$ \\
\hline $\mathrm{H} 25 \mathrm{~A}$ & 0.2829 & 1.1940 & 0.2026 & $0.080 *$ & $0.442(4)$ \\
\hline $\mathrm{C} 20 \mathrm{~B}$ & 0.2903 & $1.1658(3)$ & $0.19873(14)$ & $0.0672(18)$ & $0.558(4)$ \\
\hline $\mathrm{H} 24 \mathrm{~B}$ & 0.3151 & 1.1359 & 0.2029 & $0.081 *$ & $0.558(4)$ \\
\hline $\mathrm{H} 25 \mathrm{~B}$ & 0.3294 & 1.2159 & 0.1919 & $0.081 *$ & $0.558(4)$ \\
\hline $\mathrm{C} 21 \mathrm{~A}$ & $0.1690(4)$ & 1.1208 (4) & 0.19379 (14) & 0.0533 (19) & $0.442(4)$ \\
\hline $\mathrm{H} 26 \mathrm{~A}$ & 0.1203 & 1.1055 & 0.2029 & $0.064^{*}$ & $0.442(4)$ \\
\hline $\mathrm{H} 27 \mathrm{~A}$ & 0.1720 & 1.1514 & 0.1780 & $0.064^{*}$ & $0.442(4)$ \\
\hline $\mathrm{C} 21 \mathrm{~B}$ & $0.2294(3)$ & $1.1248(3)$ & $0.17762(11)$ & $0.0516(14)$ & $0.558(4)$ \\
\hline H26B & 0.2070 & 1.1568 & 0.1730 & $0.062 *$ & $0.558(4)$ \\
\hline H27B & 0.2540 & 1.1196 & 0.1614 & $0.062 *$ & $0.558(4)$ \\
\hline N1 & $0.06247(13)$ & $0.94267(14)$ & $0.12836(5)$ & $0.0520(6)$ & \\
\hline N2 & $0.10931(13)$ & $1.01127(13)$ & $0.16578(5)$ & $0.0481(6)$ & \\
\hline $\mathrm{Yb}$ & 0.0000 & 1.0000 & $0.14748(2)$ & $0.04418(9)$ & \\
\hline
\end{tabular}

Atomic displacement parameters $\left(\AA^{2}\right)$

\begin{tabular}{lllllll}
\hline & $U^{11}$ & $U^{22}$ & $U^{33}$ & $U^{12}$ & $U^{13}$ & $U^{23}$ \\
\hline C1 & $0.0332(15)$ & $0.0310(14)$ & $0.080(2)$ & $0.0142(12)$ & $0.0002(14)$ & $0.0028(14)$ \\
C2 & $0.0374(16)$ & $0.0342(16)$ & $0.091(2)$ & $0.0153(13)$ & $-0.0050(16)$ & $-0.0062(15)$ \\
C3 & $0.0392(17)$ & $0.0312(15)$ & $0.096(2)$ & $0.0146(13)$ & $-0.0032(16)$ & $-0.0028(15)$ \\
C4 & $0.0349(15)$ & $0.0358(15)$ & $0.094(2)$ & $0.0166(13)$ & $-0.0007(16)$ & $-0.0010(16)$ \\
C5 & $0.0480(18)$ & $0.063(2)$ & $0.086(2)$ & $0.0308(17)$ & $-0.0031(18)$ & $-0.0010(19)$ \\
C6 & $0.050(2)$ & $0.082(3)$ & $0.101(3)$ & $0.035(2)$ & $-0.013(2)$ & $-0.006(2)$ \\
C7 & $0.044(2)$ & $0.081(3)$ & $0.139(4)$ & $0.037(2)$ & $-0.006(2)$ & $-0.009(3)$ \\
C8 & $0.058(2)$ & $0.085(3)$ & $0.121(4)$ & $0.044(2)$ & $0.005(2)$ & $-0.019(3)$ \\
C9 & $0.052(2)$ & $0.062(2)$ & $0.105(3)$ & $0.0325(18)$ & $-0.010(2)$ & $-0.015(2)$ \\
C10 & $0.0388(16)$ & $0.062(2)$ & $0.083(2)$ & $0.0258(15)$ & $-0.0023(16)$ & $-0.0217(18)$ \\
C11 & $0.070(3)$ & $0.095(3)$ & $0.086(3)$ & $0.018(2)$ & $0.015(2)$ & $-0.016(2)$ \\
C12 & $0.084(3)$ & $0.155(5)$ & $0.106(4)$ & $0.017(3)$ & $0.026(3)$ & $-0.054(4)$ \\
C13 & $0.060(3)$ & $0.167(5)$ & $0.121(4)$ & $0.024(3)$ & $0.004(3)$ & $-0.089(4)$ \\
C14 & $0.058(2)$ & $0.089(3)$ & $0.163(5)$ & $0.031(2)$ & $-0.004(3)$ & $-0.065(3)$
\end{tabular}




$\begin{array}{lllllll}\mathrm{C} 15 & 0.055(2) & 0.054(2) & 0.090(3) & 0.0210(17) & 0.0019(19) & -0.0201(19) \\ \mathrm{C} 16 \mathrm{~A} & 0.0441(17) & 0.0423(17) & 0.084(2) & 0.0221(15) & -0.0159(17) & -0.0112(16) \\ \mathrm{C} 16 \mathrm{~B} & 0.0441(17) & 0.0423(17) & 0.084(2) & 0.0221(15) & -0.0159(17) & -0.0112(16) \\ \mathrm{C} 17 \mathrm{~A} & 0.055(5) & 0.052(4) & 0.077(5) & 0.019(4) & -0.015(4) & 0.006(4) \\ \mathrm{C} 17 \mathrm{~B} & 0.054(3) & 0.044(3) & 0.070(3) & 0.006(3) & 0.007(3) & -0.003(3) \\ \mathrm{C} 18 \mathrm{~A} & 0.097(7) & 0.074(6) & 0.091(7) & 0.034(6) & -0.042(6) & 0.006(5) \\ \mathrm{C} 18 \mathrm{~B} & 0.085(6) & 0.058(5) & 0.068(4) & 0.014(6) & -0.002(5) & -0.003(4) \\ \mathrm{C} 19 \mathrm{~A} & 0.091(9) & 0.088(10) & 0.066(6) & 0.029(10) & -0.021(7) & -0.011(6) \\ \mathrm{C} 19 \mathrm{~B} & 0.066(6) & 0.051(5) & 0.078(7) & 0.010(5) & -0.014(5) & -0.010(5) \\ \mathrm{C} 20 \mathrm{~A} & 0.065(7) & 0.058(6) & 0.067(7) & 0.024(6) & -0.017(5) & -0.015(6) \\ \mathrm{C} 20 \mathrm{~B} & 0.043(3) & 0.045(3) & 0.099(5) & 0.012(3) & -0.006(3) & -0.003(3) \\ \mathrm{C} 21 \mathrm{~A} & 0.053(4) & 0.055(4) & 0.048(4) & 0.023(4) & -0.003(3) & -0.006(3) \\ \mathrm{C} 21 \mathrm{~B} & 0.044(3) & 0.035(3) & 0.067(3) & 0.014(2) & 0.002(2) & 0.003(2) \\ \mathrm{N} 1 & 0.0380(13) & 0.0476(14) & 0.0731(15) & 0.0234(11) & -0.0043(13) & -0.0122(13) \\ \mathrm{N} 2 & 0.0356(13) & 0.0367(12) & 0.0716(16) & 0.0177(11) & -0.0075(12) & -0.0059(12) \\ \mathrm{Yb} & 0.03321(10) & 0.03321(10) & 0.06610(15) & 0.01661(5) & 0.000 & 0.000\end{array}$

Geometric parameters $\left(\AA,{ }^{\circ}\right)$

\begin{tabular}{llll}
\hline $\mathrm{C} 1-\mathrm{N} 1$ & $1.321(4)$ & $\mathrm{C} 16 \mathrm{~B}-\mathrm{N} 2$ & $1.459(4)$ \\
$\mathrm{C} 1-\mathrm{N} 2$ & $1.324(4)$ & $\mathrm{C} 16 \mathrm{~B}-\mathrm{C} 17 \mathrm{~B}$ & $1.645(7)$ \\
$\mathrm{C} 1-\mathrm{C} 2$ & $1.451(4)$ & $\mathrm{C} 16 \mathrm{~B}-\mathrm{H} 17 \mathrm{~B}$ & 1.0000 \\
$\mathrm{C} 1-\mathrm{Yb}$ & $2.714(3)$ & $\mathrm{C} 17 \mathrm{~A}-\mathrm{C} 18 \mathrm{~A}$ & $1.528(11)$ \\
$\mathrm{C} 2-\mathrm{C} 3$ & $1.188(4)$ & $\mathrm{C} 17 \mathrm{~A}-\mathrm{H} 18 \mathrm{~A}$ & 0.9900 \\
$\mathrm{C} 3-\mathrm{C} 4$ & $1.436(4)$ & $\mathrm{C} 17 \mathrm{~A}-\mathrm{H} 19 \mathrm{~A}$ & 0.9900 \\
$\mathrm{C} 4-\mathrm{C} 5$ & $1.385(5)$ & $\mathrm{C} 17 \mathrm{~B}-\mathrm{C} 18 \mathrm{~B}$ & $1.526(11)$ \\
$\mathrm{C} 4-\mathrm{C} 9$ & $1.393(5)$ & $\mathrm{C} 17 \mathrm{~B}-\mathrm{H} 18 \mathrm{~B}$ & 0.9900 \\
$\mathrm{C} 5-\mathrm{C} 6$ & $1.384(5)$ & $\mathrm{C} 17 \mathrm{~B}-\mathrm{H} 19 \mathrm{~B}$ & 0.9900 \\
$\mathrm{C} 5-\mathrm{H} 1$ & 0.9500 & $\mathrm{C} 18 \mathrm{~A}-\mathrm{C} 19 \mathrm{~A}$ & $1.509(18)$ \\
$\mathrm{C} 6-\mathrm{C} 7$ & $1.372(6)$ & $\mathrm{C} 18 \mathrm{~A}-\mathrm{H} 20 \mathrm{~A}$ & 0.9900 \\
$\mathrm{C} 6-\mathrm{H} 2$ & 0.9500 & $\mathrm{C} 18 \mathrm{~A}-\mathrm{H} 21 \mathrm{~A}$ & 0.9900 \\
$\mathrm{C} 7-\mathrm{C} 8$ & $1.381(6)$ & $\mathrm{C} 18 \mathrm{~B}-\mathrm{C} 19 \mathrm{~B}$ & $1.508(15)$ \\
$\mathrm{C} 7-\mathrm{H} 3$ & $\mathrm{C} 18 \mathrm{~B}-\mathrm{H} 20 \mathrm{~B}$ & 0.9900 \\
$\mathrm{C} 8-\mathrm{C} 9$ & 1.9500 & $\mathrm{C} 18 \mathrm{~B}-\mathrm{H} 21 \mathrm{~B}$ & 0.9900 \\
$\mathrm{C} 8-\mathrm{H} 4$ & $\mathrm{C} 19 \mathrm{~A}-\mathrm{C} 20 \mathrm{~A}$ & $1.53(2)$ \\
$\mathrm{C} 9-\mathrm{H} 5$ & $\mathrm{C} 19 \mathrm{~A}-\mathrm{H} 22 \mathrm{~A}$ & 0.9900 \\
$\mathrm{C} 10-\mathrm{N} 1$ & 0.9500 & $\mathrm{C} 19 \mathrm{~A}-\mathrm{H} 23 \mathrm{~A}$ & 0.9900 \\
$\mathrm{C} 10-\mathrm{C} 11$ & $\mathrm{C} 19 \mathrm{~B}-\mathrm{C} 20 \mathrm{~B}$ & $1.511(13)$ \\
$\mathrm{C} 10-\mathrm{C} 15$ & $1.958(4)$ & $\mathrm{C} 19 \mathrm{~B}-\mathrm{H} 22 \mathrm{~B}$ & 0.9900 \\
$\mathrm{C} 10-\mathrm{H} 6$ & $1.476(6)$ & $\mathrm{C} 19 \mathrm{~B}-\mathrm{H} 23 \mathrm{~B}$ & 0.9900 \\
$\mathrm{C} 11-\mathrm{C} 12$ & $1.507(4)$ & $\mathrm{C} 20 \mathrm{~A}-\mathrm{C} 21 \mathrm{~A}$ & $1.525(12)$ \\
$\mathrm{C} 11-\mathrm{H} 8$ & 1.0000 & $\mathrm{C} 20 \mathrm{~A}-\mathrm{H} 24 \mathrm{~A}$ & 0.9900 \\
$\mathrm{C} 11-\mathrm{H} 7$ & $\mathrm{C} 20 \mathrm{~A}-\mathrm{H} 25 \mathrm{~A}$ & 0.9900 \\
$\mathrm{C} 12-\mathrm{C} 13$ & $1.536(6)$ & $\mathrm{C} 20 \mathrm{~B}-\mathrm{C} 21 \mathrm{~B}$ & $1.524(8)$ \\
$\mathrm{C} 12-\mathrm{H} 10$ & $\mathrm{C} 20 \mathrm{~B}-\mathrm{H} 24 \mathrm{~B}$ & 0.9900 \\
$\mathrm{C} 12-\mathrm{H} 9$ & $\mathrm{C} 20 \mathrm{~B}-\mathrm{H} 25 \mathrm{~B}$ & 0.9900 \\
$\mathrm{C} 13-\mathrm{C} 14$ & $\mathrm{C} 21 \mathrm{~A}-\mathrm{H} 26 \mathrm{~A}$ & 0.9900 \\
$\mathrm{C} 13-\mathrm{H} 12$ & 0.9900 & $\mathrm{C} 21 \mathrm{~A}-\mathrm{H} 27 \mathrm{~A}$ &
\end{tabular}




\begin{tabular}{|c|c|c|c|}
\hline $\mathrm{C} 13-\mathrm{H} 11$ & 0.9900 & $\mathrm{C} 21 \mathrm{~B}-\mathrm{H} 26 \mathrm{~B}$ & 0.9900 \\
\hline $\mathrm{C} 14-\mathrm{C} 15$ & $1.520(5)$ & $\mathrm{C} 21 \mathrm{~B}-\mathrm{H} 27 \mathrm{~B}$ & 0.9900 \\
\hline $\mathrm{C} 14-\mathrm{H} 13$ & 0.9900 & $\mathrm{~N} 1-\mathrm{Yb}$ & $2.320(2)$ \\
\hline C14-H14 & 0.9900 & $\mathrm{~N} 2-\mathrm{Yb}$ & $2.310(2)$ \\
\hline $\mathrm{C} 15-\mathrm{H} 15$ & 0.9900 & $\mathrm{Yb}-\mathrm{N} 2^{\mathrm{i}}$ & $2.310(2)$ \\
\hline $\mathrm{C} 15-\mathrm{H} 16$ & 0.9900 & $\mathrm{Yb}-\mathrm{N} 2^{\mathrm{ii}}$ & $2.310(2)$ \\
\hline $\mathrm{C} 16 \mathrm{~A}-\mathrm{C} 17 \mathrm{~A}$ & $1.398(8)$ & $\mathrm{Yb}-\mathrm{N} 1^{\mathrm{i}}$ & $2.320(2)$ \\
\hline $\mathrm{C} 16 \mathrm{~A}-\mathrm{N} 2$ & $1.459(4)$ & $\mathrm{Yb}-\mathrm{N} 1^{\mathrm{ii}}$ & $2.320(2)$ \\
\hline $\mathrm{C} 16 \mathrm{~A}-\mathrm{C} 21 \mathrm{~A}$ & $1.477(8)$ & $\mathrm{Yb}-\mathrm{C} 1^{\mathrm{ii}}$ & $2.714(3)$ \\
\hline $\mathrm{C} 16 \mathrm{~A}-\mathrm{H} 17 \mathrm{~A}$ & 1.0000 & $\mathrm{Yb}-\mathrm{C} 1^{\mathrm{i}}$ & $2.714(3)$ \\
\hline $\mathrm{C} 16 \mathrm{~B}-\mathrm{C} 21 \mathrm{~B}$ & $1.412(6)$ & & \\
\hline $\mathrm{N} 1-\mathrm{C} 1-\mathrm{N} 2$ & $116.7(3)$ & $\mathrm{C} 19 \mathrm{~A}-\mathrm{C} 18 \mathrm{~A}-\mathrm{H} 21 \mathrm{~A}$ & 109.7 \\
\hline $\mathrm{N} 1-\mathrm{C} 1-\mathrm{C} 2$ & $122.6(3)$ & $\mathrm{C} 17 \mathrm{~A}-\mathrm{C} 18 \mathrm{~A}-\mathrm{H} 21 \mathrm{~A}$ & 109.7 \\
\hline $\mathrm{N} 2-\mathrm{C} 1-\mathrm{C} 2$ & $120.6(3)$ & $\mathrm{H} 20 \mathrm{~A}-\mathrm{C} 18 \mathrm{~A}-\mathrm{H} 21 \mathrm{~A}$ & 108.2 \\
\hline $\mathrm{N} 1-\mathrm{C} 1-\mathrm{Yb}$ & $58.68(15)$ & $\mathrm{C} 19 \mathrm{~B}-\mathrm{C} 18 \mathrm{~B}-\mathrm{C} 17 \mathrm{~B}$ & $109.9(7)$ \\
\hline $\mathrm{N} 2-\mathrm{C} 1-\mathrm{Yb}$ & $58.25(15)$ & $\mathrm{C} 19 \mathrm{~B}-\mathrm{C} 18 \mathrm{~B}-\mathrm{H} 20 \mathrm{~B}$ & 109.7 \\
\hline $\mathrm{C} 2-\mathrm{C} 1-\mathrm{Yb}$ & $174.4(2)$ & $\mathrm{C} 17 \mathrm{~B}-\mathrm{C} 18 \mathrm{~B}-\mathrm{H} 20 \mathrm{~B}$ & 109.7 \\
\hline $\mathrm{C} 3-\mathrm{C} 2-\mathrm{C} 1$ & $175.2(3)$ & $\mathrm{C} 19 \mathrm{~B}-\mathrm{C} 18 \mathrm{~B}-\mathrm{H} 21 \mathrm{~B}$ & 109.7 \\
\hline $\mathrm{C} 2-\mathrm{C} 3-\mathrm{C} 4$ & $176.7(4)$ & $\mathrm{C} 17 \mathrm{~B}-\mathrm{C} 18 \mathrm{~B}-\mathrm{H} 21 \mathrm{~B}$ & 109.7 \\
\hline $\mathrm{C} 5-\mathrm{C} 4-\mathrm{C} 9$ & $119.4(3)$ & $\mathrm{H} 20 \mathrm{~B}-\mathrm{C} 18 \mathrm{~B}-\mathrm{H} 21 \mathrm{~B}$ & 108.2 \\
\hline $\mathrm{C} 5-\mathrm{C} 4-\mathrm{C} 3$ & $119.6(3)$ & $\mathrm{C} 18 \mathrm{~A}-\mathrm{C} 19 \mathrm{~A}-\mathrm{C} 20 \mathrm{~A}$ & $111.7(10)$ \\
\hline $\mathrm{C} 9-\mathrm{C} 4-\mathrm{C} 3$ & $120.9(3)$ & $\mathrm{C} 18 \mathrm{~A}-\mathrm{C} 19 \mathrm{~A}-\mathrm{H} 22 \mathrm{~A}$ & 109.3 \\
\hline $\mathrm{C} 6-\mathrm{C} 5-\mathrm{C} 4$ & $120.5(4)$ & $\mathrm{C} 20 \mathrm{~A}-\mathrm{C} 19 \mathrm{~A}-\mathrm{H} 22 \mathrm{~A}$ & 109.3 \\
\hline $\mathrm{C} 6-\mathrm{C} 5-\mathrm{H} 1$ & 119.8 & $\mathrm{C} 18 \mathrm{~A}-\mathrm{C} 19 \mathrm{~A}-\mathrm{H} 23 \mathrm{~A}$ & 109.3 \\
\hline $\mathrm{C} 4-\mathrm{C} 5-\mathrm{H} 1$ & 119.8 & $\mathrm{C} 20 \mathrm{~A}-\mathrm{C} 19 \mathrm{~A}-\mathrm{H} 23 \mathrm{~A}$ & 109.3 \\
\hline $\mathrm{C} 7-\mathrm{C} 6-\mathrm{C} 5$ & $119.7(4)$ & $\mathrm{H} 22 \mathrm{~A}-\mathrm{C} 19 \mathrm{~A}-\mathrm{H} 23 \mathrm{~A}$ & 107.9 \\
\hline $\mathrm{C} 7-\mathrm{C} 6-\mathrm{H} 2$ & 120.2 & $\mathrm{C} 18 \mathrm{~B}-\mathrm{C} 19 \mathrm{~B}-\mathrm{C} 20 \mathrm{~B}$ & $112.3(8)$ \\
\hline $\mathrm{C} 5-\mathrm{C} 6-\mathrm{H} 2$ & 120.2 & $\mathrm{C} 18 \mathrm{~B}-\mathrm{C} 19 \mathrm{~B}-\mathrm{H} 22 \mathrm{~B}$ & 109.1 \\
\hline $\mathrm{C} 6-\mathrm{C} 7-\mathrm{C} 8$ & $120.5(4)$ & $\mathrm{C} 20 \mathrm{~B}-\mathrm{C} 19 \mathrm{~B}-\mathrm{H} 22 \mathrm{~B}$ & 109.1 \\
\hline $\mathrm{C} 6-\mathrm{C} 7-\mathrm{H} 3$ & 119.7 & $\mathrm{C} 18 \mathrm{~B}-\mathrm{C} 19 \mathrm{~B}-\mathrm{H} 23 \mathrm{~B}$ & 109.1 \\
\hline $\mathrm{C} 8-\mathrm{C} 7-\mathrm{H} 3$ & 119.7 & $\mathrm{C} 20 \mathrm{~B}-\mathrm{C} 19 \mathrm{~B}-\mathrm{H} 23 \mathrm{~B}$ & 109.1 \\
\hline $\mathrm{C} 9-\mathrm{C} 8-\mathrm{C} 7$ & $120.2(4)$ & $\mathrm{H} 22 \mathrm{~B}-\mathrm{C} 19 \mathrm{~B}-\mathrm{H} 23 \mathrm{~B}$ & 107.9 \\
\hline $\mathrm{C} 9-\mathrm{C} 8-\mathrm{H} 4$ & 119.9 & $\mathrm{C} 21 \mathrm{~A}-\mathrm{C} 20 \mathrm{~A}-\mathrm{C} 19 \mathrm{~A}$ & $108.8(10)$ \\
\hline $\mathrm{C} 7-\mathrm{C} 8-\mathrm{H} 4$ & 119.9 & $\mathrm{C} 21 \mathrm{~A}-\mathrm{C} 20 \mathrm{~A}-\mathrm{H} 24 \mathrm{~A}$ & 109.9 \\
\hline $\mathrm{C} 8-\mathrm{C} 9-\mathrm{C} 4$ & $119.7(4)$ & $\mathrm{C} 19 \mathrm{~A}-\mathrm{C} 20 \mathrm{~A}-\mathrm{H} 24 \mathrm{~A}$ & 109.9 \\
\hline $\mathrm{C} 8-\mathrm{C} 9-\mathrm{H} 5$ & 120.2 & $\mathrm{C} 21 \mathrm{~A}-\mathrm{C} 20 \mathrm{~A}-\mathrm{H} 25 \mathrm{~A}$ & 109.9 \\
\hline $\mathrm{C} 4-\mathrm{C} 9-\mathrm{H} 5$ & 120.2 & $\mathrm{C} 19 \mathrm{~A}-\mathrm{C} 20 \mathrm{~A}-\mathrm{H} 25 \mathrm{~A}$ & 109.9 \\
\hline $\mathrm{N} 1-\mathrm{C} 10-\mathrm{C} 11$ & $112.1(3)$ & $\mathrm{H} 24 \mathrm{~A}-\mathrm{C} 20 \mathrm{~A}-\mathrm{H} 25 \mathrm{~A}$ & 108.3 \\
\hline $\mathrm{N} 1-\mathrm{C} 10-\mathrm{C} 15$ & $109.9(3)$ & $\mathrm{C} 19 \mathrm{~B}-\mathrm{C} 20 \mathrm{~B}-\mathrm{C} 21 \mathrm{~B}$ & $110.1(6)$ \\
\hline $\mathrm{C} 11-\mathrm{C} 10-\mathrm{C} 15$ & $111.3(3)$ & $\mathrm{C} 19 \mathrm{~B}-\mathrm{C} 20 \mathrm{~B}-\mathrm{H} 24 \mathrm{~B}$ & 109.6 \\
\hline $\mathrm{N} 1-\mathrm{C} 10-\mathrm{H} 6$ & 107.8 & $\mathrm{C} 21 \mathrm{~B}-\mathrm{C} 20 \mathrm{~B}-\mathrm{H} 24 \mathrm{~B}$ & 109.6 \\
\hline $\mathrm{C} 11-\mathrm{C} 10-\mathrm{H} 6$ & 107.8 & $\mathrm{C} 19 \mathrm{~B}-\mathrm{C} 20 \mathrm{~B}-\mathrm{H} 25 \mathrm{~B}$ & 109.6 \\
\hline $\mathrm{C} 15-\mathrm{C} 10-\mathrm{H} 6$ & 107.8 & $\mathrm{C} 21 \mathrm{~B}-\mathrm{C} 20 \mathrm{~B}-\mathrm{H} 25 \mathrm{~B}$ & 109.6 \\
\hline $\mathrm{C} 10-\mathrm{C} 11-\mathrm{C} 12$ & $111.1(4)$ & $\mathrm{H} 24 \mathrm{~B}-\mathrm{C} 20 \mathrm{~B}-\mathrm{H} 25 \mathrm{~B}$ & 108.1 \\
\hline $\mathrm{C} 10-\mathrm{C} 11-\mathrm{H} 8$ & 109.4 & $\mathrm{C} 16 \mathrm{~A}-\mathrm{C} 21 \mathrm{~A}-\mathrm{C} 20 \mathrm{~A}$ & $112.0(7)$ \\
\hline $\mathrm{C} 12-\mathrm{C} 11-\mathrm{H} 8$ & 109.4 & $\mathrm{C} 16 \mathrm{~A}-\mathrm{C} 21 \mathrm{~A}-\mathrm{H} 26 \mathrm{~A}$ & 109.2 \\
\hline $\mathrm{C} 10-\mathrm{C} 11-\mathrm{H} 7$ & 109.4 & $\mathrm{C} 20 \mathrm{~A}-\mathrm{C} 21 \mathrm{~A}-\mathrm{H} 26 \mathrm{~A}$ & 109.2 \\
\hline
\end{tabular}




\begin{tabular}{|c|c|c|c|}
\hline $\mathrm{C} 12-\mathrm{C} 11-\mathrm{H} 7$ & 109.4 & $\mathrm{C} 16 \mathrm{~A}-\mathrm{C} 21 \mathrm{~A}-\mathrm{H} 27 \mathrm{~A}$ & 109.2 \\
\hline $\mathrm{H} 8-\mathrm{C} 11-\mathrm{H} 7$ & 108.0 & $\mathrm{C} 20 \mathrm{~A}-\mathrm{C} 21 \mathrm{~A}-\mathrm{H} 27 \mathrm{~A}$ & 109.2 \\
\hline $\mathrm{C} 13-\mathrm{C} 12-\mathrm{C} 11$ & $109.9(4)$ & $\mathrm{H} 26 \mathrm{~A}-\mathrm{C} 21 \mathrm{~A}-\mathrm{H} 27 \mathrm{~A}$ & 107.9 \\
\hline $\mathrm{C} 13-\mathrm{C} 12-\mathrm{H} 10$ & 109.7 & $\mathrm{C} 16 \mathrm{~B}-\mathrm{C} 21 \mathrm{~B}-\mathrm{C} 20 \mathrm{~B}$ & $115.1(5)$ \\
\hline $\mathrm{C} 11-\mathrm{C} 12-\mathrm{H} 10$ & 109.7 & $\mathrm{C} 16 \mathrm{~B}-\mathrm{C} 21 \mathrm{~B}-\mathrm{H} 26 \mathrm{~B}$ & 108.5 \\
\hline $\mathrm{C} 13-\mathrm{C} 12-\mathrm{H} 9$ & 109.7 & $\mathrm{C} 20 \mathrm{~B}-\mathrm{C} 21 \mathrm{~B}-\mathrm{H} 26 \mathrm{~B}$ & 108.5 \\
\hline $\mathrm{C} 11-\mathrm{C} 12-\mathrm{H} 9$ & 109.7 & $\mathrm{C} 16 \mathrm{~B}-\mathrm{C} 21 \mathrm{~B}-\mathrm{H} 27 \mathrm{~B}$ & 108.5 \\
\hline $\mathrm{H} 10-\mathrm{C} 12-\mathrm{H} 9$ & 108.2 & $\mathrm{C} 20 \mathrm{~B}-\mathrm{C} 21 \mathrm{~B}-\mathrm{H} 27 \mathrm{~B}$ & 108.5 \\
\hline $\mathrm{C} 14-\mathrm{C} 13-\mathrm{C} 12$ & $110.7(4)$ & $\mathrm{H} 26 \mathrm{~B}-\mathrm{C} 21 \mathrm{~B}-\mathrm{H} 27 \mathrm{~B}$ & 107.5 \\
\hline $\mathrm{C} 14-\mathrm{C} 13-\mathrm{H} 12$ & 109.5 & $\mathrm{C} 1-\mathrm{N} 1-\mathrm{C} 10$ & $120.5(3)$ \\
\hline $\mathrm{C} 12-\mathrm{C} 13-\mathrm{H} 12$ & 109.5 & $\mathrm{C} 1-\mathrm{N} 1-\mathrm{Yb}$ & $92.22(18)$ \\
\hline $\mathrm{C} 14-\mathrm{C} 13-\mathrm{H} 11$ & 109.5 & $\mathrm{C} 10-\mathrm{N} 1-\mathrm{Yb}$ & $147.1(2)$ \\
\hline $\mathrm{C} 12-\mathrm{C} 13-\mathrm{H} 11$ & 109.5 & $\mathrm{C} 1-\mathrm{N} 2-\mathrm{C} 16 \mathrm{~B}$ & $120.6(2)$ \\
\hline $\mathrm{H} 12-\mathrm{C} 13-\mathrm{H} 11$ & 108.1 & $\mathrm{C} 1-\mathrm{N} 2-\mathrm{C} 16 \mathrm{~A}$ & $120.6(2)$ \\
\hline $\mathrm{C} 13-\mathrm{C} 14-\mathrm{C} 15$ & $111.2(4)$ & $\mathrm{C} 1-\mathrm{N} 2-\mathrm{Yb}$ & $92.59(18)$ \\
\hline $\mathrm{C} 13-\mathrm{C} 14-\mathrm{H} 13$ & 109.4 & $\mathrm{C} 16 \mathrm{~B}-\mathrm{N} 2-\mathrm{Yb}$ & $145.21(19)$ \\
\hline $\mathrm{C} 15-\mathrm{C} 14-\mathrm{H} 13$ & 109.4 & $\mathrm{C} 16 \mathrm{~A}-\mathrm{N} 2-\mathrm{Yb}$ & $145.21(19)$ \\
\hline $\mathrm{C} 13-\mathrm{C} 14-\mathrm{H} 14$ & 109.4 & $\mathrm{~N} 2^{\mathrm{i}}-\mathrm{Yb}-\mathrm{N} 2^{\mathrm{ii}}$ & $105.17(7)$ \\
\hline $\mathrm{C} 15-\mathrm{C} 14-\mathrm{H} 14$ & 109.4 & $\mathrm{~N} 2 \mathrm{i}-\mathrm{Yb}-\mathrm{N} 2$ & $105.17(7)$ \\
\hline $\mathrm{H} 13-\mathrm{C} 14-\mathrm{H} 14$ & 108.0 & $\mathrm{~N} 2{ }^{\mathrm{ii}}-\mathrm{Yb}-\mathrm{N} 2$ & $105.17(7)$ \\
\hline $\mathrm{C} 10-\mathrm{C} 15-\mathrm{C} 14$ & $111.8(3)$ & $\mathrm{N} 2^{\mathrm{i}}-\mathrm{Yb}-\mathrm{N} 1^{\mathrm{i}}$ & $58.20(9)$ \\
\hline $\mathrm{C} 10-\mathrm{C} 15-\mathrm{H} 15$ & 109.3 & $\mathrm{~N} 2^{\mathrm{ii}}-\mathrm{Yb}-\mathrm{N} 1^{\mathrm{i}}$ & $156.22(8)$ \\
\hline $\mathrm{C} 14-\mathrm{C} 15-\mathrm{H} 15$ & 109.3 & $\mathrm{~N} 2-\mathrm{Yb}-\mathrm{N} 1^{\mathrm{i}}$ & $96.18(9)$ \\
\hline $\mathrm{C} 10-\mathrm{C} 15-\mathrm{H} 16$ & 109.3 & $\mathrm{~N} 2^{\mathrm{i}}-\mathrm{Yb}-\mathrm{N} 1^{\mathrm{ii}}$ & $96.18(9)$ \\
\hline $\mathrm{C} 14-\mathrm{C} 15-\mathrm{H} 16$ & 109.3 & $\mathrm{~N} 2^{\mathrm{ii}}-\mathrm{Yb}-\mathrm{N} 1^{\mathrm{ii}}$ & $58.19(9)$ \\
\hline $\mathrm{H} 15-\mathrm{C} 15-\mathrm{H} 16$ & 107.9 & $\mathrm{~N} 2-\mathrm{Yb}-\mathrm{N} 1^{\mathrm{ii}}$ & $156.22(8)$ \\
\hline $\mathrm{C} 17 \mathrm{~A}-\mathrm{C} 16 \mathrm{~A}-\mathrm{N} 2$ & $115.6(4)$ & $\mathrm{N} 1^{\mathrm{i}}-\mathrm{Yb}-\mathrm{N} 1^{\mathrm{ii}}$ & $104.02(8)$ \\
\hline $\mathrm{C} 17 \mathrm{~A}-\mathrm{C} 16 \mathrm{~A}-\mathrm{C} 21 \mathrm{~A}$ & $119.3(5)$ & $\mathrm{N} 2 \mathrm{i}-\mathrm{Yb}-\mathrm{N} 1$ & $156.22(8)$ \\
\hline $\mathrm{N} 2-\mathrm{C} 16 \mathrm{~A}-\mathrm{C} 21 \mathrm{~A}$ & $109.1(3)$ & $\mathrm{N} 2^{2 i}-\mathrm{Yb}-\mathrm{N} 1$ & $96.18(8)$ \\
\hline $\mathrm{C} 17 \mathrm{~A}-\mathrm{C} 16 \mathrm{~A}-\mathrm{H} 17 \mathrm{~A}$ & 103.5 & $\mathrm{~N} 2-\mathrm{Yb}-\mathrm{N} 1$ & $58.19(9)$ \\
\hline $\mathrm{N} 2-\mathrm{C} 16 \mathrm{~A}-\mathrm{H} 17 \mathrm{~A}$ & 103.5 & $\mathrm{~N} 1 \mathrm{i}-\mathrm{Yb}-\mathrm{N} 1$ & $104.02(8)$ \\
\hline $\mathrm{C} 21 \mathrm{~A}-\mathrm{C} 16 \mathrm{~A}-\mathrm{H} 17 \mathrm{~A}$ & 103.5 & 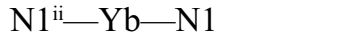 & $104.02(8)$ \\
\hline $\mathrm{C} 21 \mathrm{~B}-\mathrm{C} 16 \mathrm{~B}-\mathrm{N} 2$ & $117.4(4)$ & $\mathrm{N} 2^{\mathrm{i}}-\mathrm{Yb}-\mathrm{C} 1^{\mathrm{ii}}$ & $103.62(8)$ \\
\hline $\mathrm{C} 21 \mathrm{~B}-\mathrm{C} 16 \mathrm{~B}-\mathrm{C} 17 \mathrm{~B}$ & $107.5(4)$ & $\mathrm{N} 2^{\mathrm{ii}}-\mathrm{Yb}-\mathrm{C} 1^{\mathrm{ii}}$ & $29.16(9)$ \\
\hline $\mathrm{N} 2-\mathrm{C} 16 \mathrm{~B}-\mathrm{C} 17 \mathrm{~B}$ & $108.2(3)$ & $\mathrm{N} 2-\mathrm{Yb}-\mathrm{C} 1^{\mathrm{ii}}$ & $131.74(9)$ \\
\hline $\mathrm{C} 21 \mathrm{~B}-\mathrm{C} 16 \mathrm{~B}-\mathrm{H} 17 \mathrm{~B}$ & 107.8 & 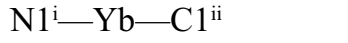 & $131.99(9)$ \\
\hline N2-C16B-H17B & 107.8 & $\mathrm{~N} 1^{\mathrm{ii}}-\mathrm{Yb}-\mathrm{C} 1^{\mathrm{ii}}$ & $29.10(9)$ \\
\hline $\mathrm{C} 17 \mathrm{~B}-\mathrm{C} 16 \mathrm{~B}-\mathrm{H} 17 \mathrm{~B}$ & 107.8 & $\mathrm{~N} 1-\mathrm{Yb}-\mathrm{C} 1^{\mathrm{ii}}$ & $100.12(8)$ \\
\hline $\mathrm{C} 16 \mathrm{~A}-\mathrm{C} 17 \mathrm{~A}-\mathrm{C} 18 \mathrm{~A}$ & $112.0(6)$ & $\mathrm{N} 2^{\mathrm{i}}-\mathrm{Yb}-\mathrm{C} 1^{\mathrm{i}}$ & $29.16(9)$ \\
\hline $\mathrm{C} 16 \mathrm{~A}-\mathrm{C} 17 \mathrm{~A}-\mathrm{H} 18 \mathrm{~A}$ & 109.2 & $\mathrm{~N} 2^{\mathrm{ii}-}-\mathrm{Yb}-\mathrm{C} 1^{\mathrm{i}}$ & $131.74(9)$ \\
\hline $\mathrm{C} 18 \mathrm{~A}-\mathrm{C} 17 \mathrm{~A}-\mathrm{H} 18 \mathrm{~A}$ & 109.2 & $\mathrm{~N} 2-\mathrm{Yb}-\mathrm{C} 1^{\mathrm{i}}$ & $103.62(8)$ \\
\hline $\mathrm{C} 16 \mathrm{~A}-\mathrm{C} 17 \mathrm{~A}-\mathrm{H} 19 \mathrm{~A}$ & 109.2 & $\mathrm{~N} 1^{\mathrm{i}}-\mathrm{Yb}-\mathrm{C} 1^{\mathrm{i}}$ & $29.10(9)$ \\
\hline $\mathrm{C} 18 \mathrm{~A}-\mathrm{C} 17 \mathrm{~A}-\mathrm{H} 19 \mathrm{~A}$ & 109.2 & $\mathrm{~N} 1^{\mathrm{ii}}-\mathrm{Yb}-\mathrm{C} 1^{\mathrm{i}}$ & $100.12(8)$ \\
\hline $\mathrm{H} 18 \mathrm{~A}-\mathrm{C} 17 \mathrm{~A}-\mathrm{H} 19 \mathrm{~A}$ & 107.9 & $\mathrm{~N} 1-\mathrm{Yb}-\mathrm{C} 1^{\mathrm{i}}$ & $131.99(9)$ \\
\hline $\mathrm{C} 18 \mathrm{~B}-\mathrm{C} 17 \mathrm{~B}-\mathrm{C} 16 \mathrm{~B}$ & $112.2(6)$ & $\mathrm{C} 1^{\mathrm{ii}-}-\mathrm{Yb}-\mathrm{C} 1^{\mathrm{i}}$ & $119.946(6)$ \\
\hline $\mathrm{C} 18 \mathrm{~B}-\mathrm{C} 17 \mathrm{~B}-\mathrm{H} 18 \mathrm{~B}$ & 109.2 & $\mathrm{~N} 2 \mathrm{i}-\mathrm{Yb}-\mathrm{C} 1$ & $131.74(9)$ \\
\hline $\mathrm{C} 16 \mathrm{~B}-\mathrm{C} 17 \mathrm{~B}-\mathrm{H} 18 \mathrm{~B}$ & 109.2 & $\mathrm{~N} 2{ }^{\mathrm{ii}}-\mathrm{Yb}-\mathrm{C} 1$ & $103.62(8)$ \\
\hline $\mathrm{C} 18 \mathrm{~B}-\mathrm{C} 17 \mathrm{~B}-\mathrm{H} 19 \mathrm{~B}$ & 109.2 & $\mathrm{~N} 2-\mathrm{Yb}-\mathrm{C} 1$ & $29.16(9)$ \\
\hline
\end{tabular}




\begin{tabular}{|c|c|}
\hline $\mathrm{C} 16 \mathrm{~B}-\mathrm{C} 17 \mathrm{~B}-\mathrm{H} 19 \mathrm{~B}$ & 109.2 \\
\hline $\mathrm{H} 18 \mathrm{~B}-\mathrm{C} 17 \mathrm{~B}-\mathrm{H} 19 \mathrm{~B}$ & 107.9 \\
\hline $\mathrm{C} 19 \mathrm{~A}-\mathrm{C} 18 \mathrm{~A}-\mathrm{C} 17 \mathrm{~A}$ & $110.0(8)$ \\
\hline $\mathrm{C} 19 \mathrm{~A}-\mathrm{C} 18 \mathrm{~A}-\mathrm{H} 20 \mathrm{~A}$ & 109.7 \\
\hline $\mathrm{C} 17 \mathrm{~A}-\mathrm{C} 18 \mathrm{~A}-\mathrm{H} 20 \mathrm{~A}$ & 109.7 \\
\hline $\mathrm{C} 9-\mathrm{C} 4-\mathrm{C} 5-\mathrm{C} 6$ & $-1.4(5)$ \\
\hline $\mathrm{C} 3-\mathrm{C} 4-\mathrm{C} 5-\mathrm{C} 6$ & $-178.4(3)$ \\
\hline $\mathrm{C} 4-\mathrm{C} 5-\mathrm{C} 6-\mathrm{C} 7$ & $-0.2(6)$ \\
\hline $\mathrm{C} 5-\mathrm{C} 6-\mathrm{C} 7-\mathrm{C} 8$ & $1.7(7)$ \\
\hline $\mathrm{C} 6-\mathrm{C} 7-\mathrm{C} 8-\mathrm{C} 9$ & $-1.7(7)$ \\
\hline $\mathrm{C} 7-\mathrm{C} 8-\mathrm{C} 9-\mathrm{C} 4$ & $0.0(6)$ \\
\hline $\mathrm{C} 5-\mathrm{C} 4-\mathrm{C} 9-\mathrm{C} 8$ & $1.5(6)$ \\
\hline $\mathrm{C} 3-\mathrm{C} 4-\mathrm{C} 9-\mathrm{C} 8$ & $178.5(3)$ \\
\hline $\mathrm{N} 1-\mathrm{C} 10-\mathrm{C} 11-\mathrm{C} 12$ & $-179.1(3)$ \\
\hline $\mathrm{C} 15-\mathrm{C} 10-\mathrm{C} 11-\mathrm{C} 12$ & $-55.5(5)$ \\
\hline $\mathrm{C} 10-\mathrm{C} 11-\mathrm{C} 12-\mathrm{C} 13$ & $57.3(7)$ \\
\hline $\mathrm{C} 11-\mathrm{C} 12-\mathrm{C} 13-\mathrm{C} 14$ & $-57.7(7)$ \\
\hline $\mathrm{C} 12-\mathrm{C} 13-\mathrm{C} 14-\mathrm{C} 15$ & $56.7(6)$ \\
\hline $\mathrm{N} 1-\mathrm{C} 10-\mathrm{C} 15-\mathrm{C} 14$ & $179.0(4)$ \\
\hline $\mathrm{C} 11-\mathrm{C} 10-\mathrm{C} 15-\mathrm{C} 14$ & $54.2(5)$ \\
\hline $\mathrm{C} 13-\mathrm{C} 14-\mathrm{C} 15-\mathrm{C} 10$ & $-54.7(5)$ \\
\hline $\mathrm{N} 2-\mathrm{C} 16 \mathrm{~A}-\mathrm{C} 17 \mathrm{~A}-\mathrm{C} 18 \mathrm{~A}$ & $178.9(6)$ \\
\hline $\mathrm{C} 21 \mathrm{~A}-\mathrm{C} 16 \mathrm{~A}-\mathrm{C} 17 \mathrm{~A}-\mathrm{C} 18 \mathrm{~A}$ & $-47.8(9)$ \\
\hline $\mathrm{C} 21 \mathrm{~B}-\mathrm{C} 16 \mathrm{~B}-\mathrm{C} 17 \mathrm{~B}-\mathrm{C} 18 \mathrm{~B}$ & $-53.7(7)$ \\
\hline $\mathrm{N} 2-\mathrm{C} 16 \mathrm{~B}-\mathrm{C} 17 \mathrm{~B}-\mathrm{C} 18 \mathrm{~B}$ & $178.6(6)$ \\
\hline $\mathrm{C} 16 \mathrm{~A}-\mathrm{C} 17 \mathrm{~A}-\mathrm{C} 18 \mathrm{~A}-\mathrm{C} 19 \mathrm{~A}$ & $51.2(11)$ \\
\hline $\mathrm{C} 16 \mathrm{~B}-\mathrm{C} 17 \mathrm{~B}-\mathrm{C} 18 \mathrm{~B}-\mathrm{C} 19 \mathrm{~B}$ & $52.9(11)$ \\
\hline $\mathrm{C} 17 \mathrm{~A}-\mathrm{C} 18 \mathrm{~A}-\mathrm{C} 19 \mathrm{~A}-\mathrm{C} 20 \mathrm{~A}$ & $-57.9(14)$ \\
\hline $\mathrm{C} 17 \mathrm{~B}-\mathrm{C} 18 \mathrm{~B}-\mathrm{C} 19 \mathrm{~B}-\mathrm{C} 20 \mathrm{~B}$ & $-55.0(12)$ \\
\hline $\mathrm{C} 18 \mathrm{~A}-\mathrm{C} 19 \mathrm{~A}-\mathrm{C} 20 \mathrm{~A}-\mathrm{C} 21 \mathrm{~A}$ & $57.1(14)$ \\
\hline $\mathrm{C} 18 \mathrm{~B}-\mathrm{C} 19 \mathrm{~B}-\mathrm{C} 20 \mathrm{~B}-\mathrm{C} 21 \mathrm{~B}$ & $55.8(11)$ \\
\hline $\mathrm{C} 17 \mathrm{~A}-\mathrm{C} 16 \mathrm{~A}-\mathrm{C} 21 \mathrm{~A}-\mathrm{C} 20 \mathrm{~A}$ & $47.8(9)$ \\
\hline $\mathrm{N} 2-\mathrm{C} 16 \mathrm{~A}-\mathrm{C} 21 \mathrm{~A}-\mathrm{C} 20 \mathrm{~A}$ & $-176.2(7)$ \\
\hline $\mathrm{C} 19 \mathrm{~A}-\mathrm{C} 20 \mathrm{~A}-\mathrm{C} 21 \mathrm{~A}-\mathrm{C} 16 \mathrm{~A}$ & $-49.3(12)$ \\
\hline
\end{tabular}

\begin{tabular}{|c|c|}
\hline $\mathrm{N} 1-\mathrm{Y} b-\mathrm{C} 1$ & $100.12(8)$ \\
\hline $\mathrm{N} 1{ }^{\mathrm{ii}}-\mathrm{Yb}-\mathrm{C} 1$ & $131.98(9)$ \\
\hline $\mathrm{N} 1-\mathrm{Yb}-\mathrm{C} 1$ & $29.10(9)$ \\
\hline 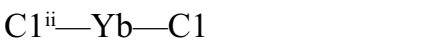 & $119.947(6)$ \\
\hline $\mathrm{C} 1 \mathrm{i}-\mathrm{Yb}-\mathrm{C} 1$ & $119.945(6)$ \\
\hline $\mathrm{N} 2-\mathrm{C} 16 \mathrm{~B}-\mathrm{C} 21 \mathrm{~B}-\mathrm{C} 20 \mathrm{~B}$ & $178.3(4)$ \\
\hline $\mathrm{C} 17 \mathrm{~B}-\mathrm{C} 16 \mathrm{~B}-\mathrm{C} 21 \mathrm{~B}-\mathrm{C} 20 \mathrm{~B}$ & $56.2(6)$ \\
\hline $\mathrm{C} 19 \mathrm{~B}-\mathrm{C} 20 \mathrm{~B}-\mathrm{C} 21 \mathrm{~B}-\mathrm{C} 16 \mathrm{~B}$ & $-59.6(8)$ \\
\hline $\mathrm{N} 2-\mathrm{C} 1-\mathrm{N} 1-\mathrm{C} 10$ & $-171.7(3)$ \\
\hline $\mathrm{C} 2-\mathrm{C} 1-\mathrm{N} 1-\mathrm{C} 10$ & $9.7(4)$ \\
\hline $\mathrm{Yb}-\mathrm{C} 1-\mathrm{N} 1-\mathrm{C} 10$ & $-176.7(3)$ \\
\hline $\mathrm{N} 2-\mathrm{C} 1-\mathrm{N} 1-\mathrm{Yb}$ & $4.9(3)$ \\
\hline $\mathrm{C} 2-\mathrm{C} 1-\mathrm{N} 1-\mathrm{Yb}$ & $-173.6(3)$ \\
\hline $\mathrm{C} 11-\mathrm{C} 10-\mathrm{N} 1-\mathrm{C} 1$ & $-104.2(4)$ \\
\hline $\mathrm{C} 15-\mathrm{C} 10-\mathrm{N} 1-\mathrm{C} 1$ & $131.5(3)$ \\
\hline $\mathrm{C} 11-\mathrm{C} 10-\mathrm{N} 1-\mathrm{Yb}$ & $81.9(5)$ \\
\hline $\mathrm{C} 15-\mathrm{C} 10-\mathrm{N} 1-\mathrm{Yb}$ & $-42.4(6)$ \\
\hline $\mathrm{N} 1-\mathrm{C} 1-\mathrm{N} 2-\mathrm{C} 16 \mathrm{~B}$ & $-174.1(3)$ \\
\hline $\mathrm{C} 2-\mathrm{C} 1-\mathrm{N} 2-\mathrm{C} 16 \mathrm{~B}$ & $4.5(4)$ \\
\hline $\mathrm{Yb}-\mathrm{C} 1-\mathrm{N} 2-\mathrm{C} 16 \mathrm{~B}$ & $-169.2(3)$ \\
\hline $\mathrm{N} 1-\mathrm{C} 1-\mathrm{N} 2-\mathrm{C} 16 \mathrm{~A}$ & $-174.1(3)$ \\
\hline $\mathrm{C} 2-\mathrm{C} 1-\mathrm{N} 2-\mathrm{C} 16 \mathrm{~A}$ & $4.5(4)$ \\
\hline $\mathrm{Yb}-\mathrm{C} 1-\mathrm{N} 2-\mathrm{C} 16 \mathrm{~A}$ & $-169.2(3)$ \\
\hline $\mathrm{N} 1-\mathrm{C} 1-\mathrm{N} 2-\mathrm{Yb}$ & $-5.0(3)$ \\
\hline $\mathrm{C} 2-\mathrm{C} 1-\mathrm{N} 2-\mathrm{Yb}$ & $173.6(3)$ \\
\hline $\mathrm{C} 21 \mathrm{~B}-\mathrm{C} 16 \mathrm{~B}-\mathrm{N} 2-\mathrm{C} 1$ & $82.5(4)$ \\
\hline $\mathrm{C} 17 \mathrm{~B}-\mathrm{C} 16 \mathrm{~B}-\mathrm{N} 2-\mathrm{C} 1$ & $-155.7(3)$ \\
\hline $\mathrm{C} 21 \mathrm{~B}-\mathrm{C} 16 \mathrm{~B}-\mathrm{N} 2-\mathrm{Yb}$ & $-78.2(5)$ \\
\hline $\mathrm{C} 17 \mathrm{~B}-\mathrm{C} 16 \mathrm{~B}-\mathrm{N} 2-\mathrm{Yb}$ & $43.5(5)$ \\
\hline $\mathrm{C} 17 \mathrm{~A}-\mathrm{C} 16 \mathrm{~A}-\mathrm{N} 2-\mathrm{C} 1$ & $-74.5(5)$ \\
\hline $\mathrm{C} 21 \mathrm{~A}-\mathrm{C} 16 \mathrm{~A}-\mathrm{N} 2-\mathrm{C} 1$ & $147.7(4)$ \\
\hline $\mathrm{C} 17 \mathrm{~A}-\mathrm{C} 16 \mathrm{~A}-\mathrm{N} 2-\mathrm{Yb}$ & $124.7(5)$ \\
\hline $\mathrm{C} 21 \mathrm{~A}-\mathrm{C} 16 \mathrm{~A}-\mathrm{N} 2-\mathrm{Yb}$ & $-13.1(6)$ \\
\hline
\end{tabular}

Symmetry codes: (i) $-y+1, x-y+2, z$; (ii) $-x+y-1,-x+1, z$. 